Graphical Abstract

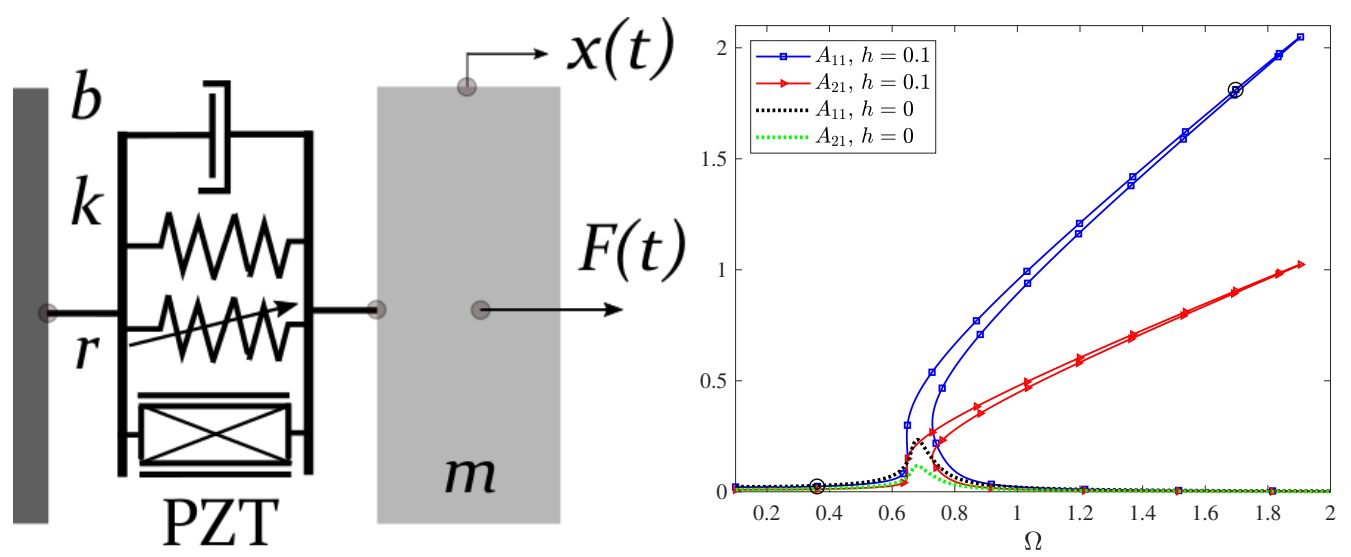




\title{
Parametrically amplified Mathieu-Duffing nonlinear energy harvesters
}

\author{
Danilo Karličić $^{\mathrm{a}, \mathrm{b}, *}$, Tanmoy Chatterjee ${ }^{\mathrm{a}}$, Milan Cajić ${ }^{\mathrm{b}}$, Sondipon Adhikari ${ }^{\mathrm{a}}$ \\ ${ }^{a}$ College of Engineering, Swansea University, United Kingdom \\ ${ }^{b}$ Mathematical institute of the Serbian Academy of Sciences and Arts, Kneza Mihaila 36, Belgrade, Serbia
}

\begin{abstract}
The steady-state response of a nonlinear piezoelectric energy harvester subjected to external and parametric excitation is investigated based on the Mathieu-Duffing nonlinear oscillator model. The parametric excitation is introduced to amplify the external harmonic excitation and extend the capabilities of the nonlinear piezoelectric energy harvester device. To obtain the approximated solution of the nonlinear periodic responses for displacement and electrical voltage of the energy harvester, the incremental harmonic balance method in combination with the path-following technique is adopted. It is assumed that the proposed nonlinear model consists of cubic and quadratic nonlinearity, where parametric amplification appears in the form of a trigonometric function. The frequency is tuned as one-to-one and the one-to-two ratio between external and parametric excitation. The effects of quadratic and cubic nonlinearity as well as parametric amplification are studied in detail, and their incredible properties to extend harvester application performance is illustrated. It is explicitly demonstrated that for some particular combination of the system parameters, vibration amplitudes and harvested power can be amplified up to three or five times in comparison to the classical broadband nonlinear energy harvester based on the forced Duffing oscillator. This extraordinary amplification shown to be a key motivation to realize the proposed concept in practice. The presence of combined quadratic and cubic nonlinearities resulted in both hardening and softening spring behavior and leading to the appearance of coexisting periodic solutions in the amplitude-frequency responses. Periodic orbits obtained by the proposed methodology are verified with the results from direct numerical integration and fine agreement is demonstrated. Moreover, a significant influence of the parametric amplification on the instantaneous power is revealed in time response diagrams, thus showing better performance of the proposed energy harvester system.
\end{abstract}

Keywords: Energy harvester, Parametric amplification, Nonlinear response, Mathieu-Duffing oscillator, Incremental harmonic balance method.

\section{Introduction}

The process of capturing small amounts of energy from one or more naturally occurring environmental energy sources such as thermal, solar, electromagnetic, and mechanical is known as energy harvesting. The energy harvester (EH) developed for capturing the ambient vibration energy by converting the mechanical strain energy into the electric current or voltage, using the piezoelectric effect, are usually called piezoelectric energy harvesters [1]. The self-powering capabilities of piezoelectric structures allow electronic devices as wireless sensors to exclude energy storage components and retains promise for direct driving of energy from the environmental mechanical resources by powering small electronics and MEMS/NEMS devices [2]. Understanding the general dynamic behaviour of such electromechanical systems is of prime importance for design procedures and practical applications of EH devices. Therefore, this study aims at demonstrating a better performance of the nonlinear EH model with parametric amplification leading to higher values of the harvested power. The basic approach used in design of EH devices is tuning of the mechanical part in order to vibrate with the resonant frequency [3]. When a system is subjected to a single frequency excitation, the resonant harvesting device needs to be tuned to the excitation frequency. One of the possibilities to extend the capability of linear EH is based on multiple connected structures as shown in [4], where the mechanical part of the EH can resonate in a wide spectrum of frequencies. The main drawback of linear $\mathrm{EH}$ devices is in the fact that they are efficient only in the case when excitation frequency is close to one

* Corresponding author

Email address: danilo.karlicic@swansea.ac.uk (Danilo Karličić ) 
of the resonance frequencies. Therefore, most of the linear energy harvesting devices are designed based on the assumption that the ambient excitation has some known form, typically single frequency harmonic excitation. The efficiency of linear EH devices reduces drastically when the system is out of resonance. The potential solution to this kind of problem in the design of $\mathrm{EH}$ devices is the application of broadband $\mathrm{EH}$ devices [5] based on nonlinear properties [6], which is not critically sensitive to the particular environmental conditions such as frequency or amplitude of excitation. The main sources of nonlinearity that appear in these types of EH systems can be from the material, geometrical or inertia properties. Nonlinear EH systems relies on the use of a double [7] and higher-order potential well functions to obtain two or more equilibrium positions. The main advantage of a double potential well for energy harvesting is achieved particularly in the system with internal excitation. The simplest equation of motion with a double potential well is related to the well-known Duffing oscillator with the cubic nonlinear term, which has been extensively studied in the literature $[8,9]$. This type of nonlinearity belongs to the group of geometric nonlinearities [10]. Dynamic behaviour of such complex systems is characterized by either existing periodic and multiple periodic solutions or chaotic responses [11]. The base excited cantilever beam has been demonstrated as a practical design concept in EH devices, especially the models that exploit geometrically nonlinear behaviour as given in [12]. Other interesting phenomena in nonlinear EH systems are the internal resonance mechanism $[13,14]$ and auto-parametric resonance [15].

Recently, special attention has been paid to the multi-stable EH devices, where the researchers observe an interesting dynamical behaviour of such nonlinear systems $[16,17]$. The main advantage of such models with multiple stable potential wells is the generation of higher energy outputs over a wide range of frequencies. Zhou et al. [18] investigated a base excited nonlinear piezoelectric energy harvester with a triple-well potential induced by a magnetic field. The authors experimentally identified the equivalent nonlinear restoring force of the tri-stable oscillator as a high order polynomial and determined key system parameters of through the optimization process. Panyam and Daqaq [19] studied the lumped-parameter electromechanical model of a tri-stable vibratory $\mathrm{EH}$ that includes a restoring force with cubic and quintic nonlinearities by introducing the multiple scale technique with experimental validation. They constructed an analytical solution describing the amplitudes and stability of the intra- and inter-well dynamics of EH. Moreover, they investigated the critical bifurcation points in the frequency responses and used them to define an effective frequency bandwidth of the nonlinear EH. In [20], the nonlinear dynamic effect of asymmetry of EH potential wells and improvement of the energy harvesting capabilities for different types of excitation forces were investigated by using the harmonic balance method. Multi-stable nonlinear EH model proposed as biomorph cantilever nonlinear Euler-Bernoulli beam with soft magnetic tip and two externally fixed permanent magnets arranged in series was analysed in [21]. It was shown that for the magnetic field induced by the external magnets, the proposed nonlinear EH model can possess multi-stable potential functions in a wide spectrum ranging from monostable to penta-stable functions. Based on numerical simulations, it was further illustrated that such system exhibits interesting nonlinear phenomena, where transitions between these multi-stable states occurs through complicated bifurcation phenomena.

The parametric amplification phenomenon relies on the amplification of amplitudes of a nonlinear harmonically excited oscillator by using the parametric pumping through a single parametric excitation term $[22,23]$. This phenomenon can be exploited in various electrical and mechanical engineering systems [24]. In the scientific literature, special attention is devoted to the application of parametric amplification in design of MEMS/NEMS devices [25, 26] based on nonlinear models with quadratic, cubic or quintic nonlinearity or their combinations. In [27], the authors used the forced Mathieu-Duffing equation including the quadratic nonlinear term as a model with parametric excitation. By introducing the method of varying amplitudes, the approximate analytical steady-state solutions and corresponding stabilities are obtained and validated by the numerical integration. It is shown that by combining nonlinearity (in the form of quadratic and cubic terms) with parametric excitation could lead to different amplitude-frequency solutions, where jumps and bi-stability phenomena might appear. In [28], the authors have analysed the cantilever microbeam model with base excitation using the mathematical model based on the Mathieu-Duffing equation with frequency tuned parametric amplifiers. Moreover, by applying the method of varying amplitudes it is found that some amplitude-frequency curves collapsed in the case of detuned super-threshold parametric amplification. The theoretical predictions are validated by numerical integration and experimental testing. Sorokin [29] investigated the dynamical behaviour of a nonlinear parametric amplifier for a wide range of system parameters, especially beyond resonance. The modified method of direct separation of motion is introduced to study the steady-state amplitude, where the nonlinear parametric amplifier response reach high values, even for small amplitudes of external excitation. Dolev and Bucher [30] analysed one and two degrees of freedom 
nonlinear model based on a weak Duffing nonlinearity and parametric excitation term. By introducing the multiple scales method, they determined the steady-state amplitude in the case when parametric excitation contains two frequencies whose combination leads to larger response amplitudes. The multi-degree of freedom mechanical system with two frequency parametric amplification and electronically tunable topology is investigated in [31]. It is shown that for specific positions of the parametric excitation force and real-time topology modification it is possible to amplify specific inputs in a three degree of freedom system. Coupling between the quadratic and cubic nonlinearities with the dual-frequency parametric amplification model of the Duffing oscillator was studied in [32]. Moreover, in [33] a combination of two piezoelectric cantilever beams with magnetic tip masses having direct and parametric excitation is introduced as a model of the EH device. Two beams are coupled through the magnetic force that reduces the necessary threshold excitation level. Introducing the time delay feedback into the Mathieu-van der Pol-Duffing oscillator based on EH, it is possible to increase harvested power at some specific frequency ranges [34]. However, the practical application of parametric excitation in the design of energy harvesting devices is provided in a series of papers proposed by Jia and co-authors [35, 36].

Until this study, a detailed investigation of the parametric amplification phenomena in EH systems with mixed nonlinearities had not been undertaken. In two similar studies, the authors employed the parametric excitation in the linear model of EH system [37] and the Duffing oscillator based EH [38]. The main difference between the present and aforementioned studies lies in the investigation of the amplification effect emerging in the harmonically excited EH system with quadratic and cubic nonlinearities that is induced by the parametric excitation.

In this context, we have assessed the current state-of-the-art and found that investigation of frequency responses and stability of the nonlinear EH with parametric amplification based on incremental harmonic balance method (IHBM) in conjunction with the path-following and Floquet theory has not been reported in the literature up to now. Therefore, the main purpose of this paper is to investigate the nonlinear periodic response of the parametrically amplified piezoelectric EH based on the model given in the form of forced Mathieu-Duffing equation with two types of parametric excitation. The adopted nonlinearity is a combination of quadratic and cubic nonlinearities. The resulting performance of the combined effect of these nonlinearities can be found in many interesting real-world physical examples such as buckling, initial curvatures, or nonlinear foundations. Here, the main focus is put on the generalized model of a nonlinear piezoelectric EH given as MEMS device. The frequency of parametric excitation is tuned as oneto-one and one-to-two ratio of frequencies of external and parametric harmonic excitation for sine and cosine functions, respectively. Such combinations of excitation forces can interact, where the parametric excitation can amplify the signal from external direct excitation. Sine and cosine parametric excitations can cause effective amplification of the signal from direct harmonic excitation. However, in [35] it was demonstrated that the the system excited by sine parametric excitation with the frequency tuned by one-to-one ratio displays significant amplification of the response amplitude without satisfying the parametric resonance condition. Such amplification effect mostly occurs in systems with mixed nonlinearities, where quadratic nonlinearity plays a significant role. Applying the IHBM and path-following methods, the series of periodic responses are given in the form of frequency response curves. Both branches of periodic responses, stable and unstable, are determined by adopting the Floquet stability theory and Hsu procedure. Important aim of the proposed analyses is to reveal the level of amplification of the response amplitude and voltage. Moreover, response amplitudes of the displacement and instantaneous power in time are given to compare the results for different values of the parametric amplification parameter.

\section{Problem formulation}

Here, the model of a nonlinear energy harvester based on the Mathieu-Duffing oscillator connected with piezoelectric element is given in Fig.1. More precisely, the lumped-parameter model consists of the oscillating mass coupled with the fixed base through a linear spring (stiffness $k$ ), linear dash-pot element (damping $b$ ), a nonlinear spring of quadratic and cubic nonlinearity type and a piezoelectric element (connected to the corresponding electric circuit and denoted as PZT in Fig.1). Moreover, we assume that the mass is excited by a combination of the external harmonic $F(t)$ and parametric $p(\Omega t)$ excitation. The main reason for introducing the parametric excitation term is to amplify the signal from the external harmonic excitation. The parametric excitation term is expressed in a trigonometric form. Similar nonlinear mechanical models can represent different macro/micro-mechanical systems including base excited macro-beams [27], MEMS $[39,40]$, NEMS [41, 42] and atomic force microscopes (AFM) [43, 44]. 
The governing equation of the piezoelectric energy harvester can be expressed in the dimensionless form as

$$
\begin{gathered}
\ddot{x}+\delta \dot{x}+\omega_{0}^{2} x+\xi x^{2}+\gamma x^{3}+h p(\Omega t) x-\chi v=f \cos (\Omega t), \\
\dot{v}+\lambda v+\tilde{k} \dot{x}=0,
\end{gathered}
$$

where time derivative $(\dot{)})$ is used to represent $d(\cdot) / d t$. The dimensionless parameters are: $x=x(t)$ is the relative displacement of the point mass $m, v(t)$ is the generated voltage across the load resistance, $\delta$ is the mechanical damping, $\omega_{0}$ is the linear natural frequency parameter, $\xi$ is the quadratic nonlinear stiffness parameter, $\gamma$ is the cubic nonlinear stiffness parameter, $\chi$ is the piezoelectric coupling term in the mechanical displacement equation, $\tilde{k}$ is the piezoelectric coupling term in the electrical circuit, $h$ is the magnitude of parametric excitation, $\lambda$ is the reciprocal of time constant of the electrical circuit, $f$ and $\Omega$ are the amplitude and frequency of external excitation, respectively. The parametric excitation term $p(\Omega t)$ can take the following two forms $\sin (\Omega t)$ and $\cos (2 \Omega t)$. Many authors have studied nonlinear oscillators

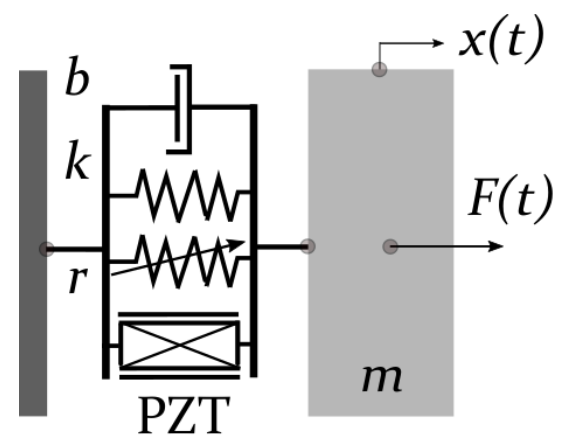

Figure 1: The lumped-parameter model of a nonlinear energy harvester with the parametric and harmonic excitation forces.

with quadratic and cubic nonlinearities investigating how to exploit and improve capabilities of EH devices. The sources of quadratic and cubic nonlinearities usually appear in the nonlinear mechanical models of cable, beam and plate structures [10,45]. As stated in [10], quadratic and cubic nonlinearities can be derived from the nonlinear governing partial differential equations of motion of a vibrating cable using the Galerkin discretisation. By varying the stiffness parameters of the quadratic and cubic nonlinearity terms, which depend on the material and geometrical parameters of a cable, one can control the influence of the nonlinearity on structural vibration. In the literature, a special attention is devoted to the analysis of the influence of quadratic nonlinearity in structural mechanical models considering slightly curved and axially loaded beams, as well as consequences of the Taylor expansion solution for beam, plate, and shell structures about buckled states of static equilibrium [45]. Also, Duffing-type nonlinearity can be used in structural mechanical models to represent mid-plane stretching and large rotations. The aforementioned nonlinear structural models can be very useful in design of nonlinear EH devices. In this work, we adopted the lumped-mass model as a generalization of the nonlinear EH model with direct and parametric excitation causing the appearance of parametric amplification phenomenon.

\section{Nonlinear periodic response}

\subsection{The incremental harmonic balance method}

The incremental harmonic balance method (IHBM) is based on the Fourier series expansion and NewtonRaphson iterative procedure proposed in [46]. This method has been subsequently reformulated by [47] to analyse higher-order periodic solutions and their bifurcations. The authors performed a case study, where bifurcation and route-to-chaos for Mathieu-Duffing oscillators were investigated by using the sub-harmonic terms in the Fourier series. Huang et al. [48] analysed the nonlinear vibrations of a curved beam subjected to uniform base harmonic excitation with two types of nonlinearities. By using the Galerkin approach and IHBM they solved the partial differential equation of motion and obtained amplitude-frequency responses. In [49], the authors suggested the incremental harmonic balance and pseudo-arc continuation technique to predict the steady-state response behaviour of a pinned-pinned beam on a nonlinear viscoelastic foundation when subjected to harmonic loads. Recently, IHBM proved to be immensely useful in determination of periodic responses and stability of highly nonlinear systems with equivalent piece-wise linearisation [50]. The main concept of the IHBM is based on few simple steps where the solution of the nonlinear system can be 
determined as the sum of the sine and cosine functions. First, introducing the dimensionless time parameter in the form of $\tau=\Omega t$ into the ordinary differential equation, and then assuming the generalized coordinates in an incremental form. The IHBM requires that the solution can be approximated by the Fourier series and application of Galerkin procedure. By applying IHBM on the system of equations Eq.(1) and Eq.(2), we obtain iterative relationships in the form of frequency increments to analyse the frequency response of the nonlinear energy harvester. One of the most important features of IHBM is simple implementation of the path-following method for evaluating the system responses corresponding to different parameters of the model. Now, by introducing the new time scale $\tau=\Omega t$, new forms of equations Eq.(1) and Eq.(2) can be obtained as

$$
\begin{gathered}
\Omega^{2} \ddot{x}+\Omega \delta \dot{x}+\omega_{0}^{2} x+\xi x^{2}+\gamma x^{3}+h p(\tau) x-\chi v=f \cos (\tau), \\
\Omega \dot{v}+\lambda v+\Omega k \dot{x}=0,
\end{gathered}
$$

where the time derivative $(\cdot)$ is used to represent $\mathrm{d}(\cdot) / \mathrm{d} \tau$. The second step is to introduce the incremental relation for generalized coordinates $x(\tau)$ and $v(\tau)$ as well as excitation frequency $\Omega$ in order to linearise the above nonlinear system. Inserting initial dynamic state of the generalized coordinates $x_{0}(\tau), v_{0}(\tau)$, excitation frequency $\Omega_{0}$ and corresponding increments $\Delta x, \Delta v$ and $\Delta \Omega$ in their neighbourhood yields

$$
x=x_{0}+\Delta x, \quad v=v_{0}+\Delta v, \quad \Omega=\Omega_{0}+\Delta \Omega .
$$

Assuming that the nonlinear system is subjected to external and parametric excitation, the solutions for $x_{0}$ and $v_{0}$ and their increments are approximated in the form of truncated Fourier series as:

$$
\begin{aligned}
& x_{0}(\tau)=a_{10}+\sum_{n=1}^{M}\left[a_{1 n} \cos (n \tau)+b_{1 n} \sin (n \tau)\right]=\mathbf{C A}_{1}, \\
& v_{0}(\tau)=a_{20}+\sum_{n=1}^{M}\left[a_{2 n} \cos (n \tau)+b_{2 n} \sin (n \tau)\right]=\mathbf{C A}_{2},
\end{aligned}
$$

where

$$
\begin{aligned}
\mathbf{C}=\left[\begin{array}{lllllllll}
1 & \cos (\tau) & \cos (2 \tau) & \ldots & \cos (n \tau) & \sin (\tau) & \sin (2 \tau) & \ldots & \sin (n \tau)
\end{array}\right] \\
\mathbf{A}_{s}=\left[\begin{array}{lllllllll}
a_{s 0} & a_{s 1} & a_{s 2} & \ldots & a_{s n} & b_{s 1} & b_{s 2} & \ldots & b_{s n}
\end{array}\right]^{T},(s=1,2) . \\
\Delta x=\mathbf{C} \Delta \mathbf{A}_{1}, \quad \Delta v=\mathbf{C} \Delta \mathbf{A}_{2},
\end{aligned}
$$

where

$$
\Delta \mathbf{A}_{s}=\left[\begin{array}{lllllllll}
\Delta a_{s 0} & \Delta a_{s 1} & \Delta a_{s 2} & \ldots & \Delta a_{s n} & \Delta b_{s 1} & \Delta b_{s 2} & \ldots & \Delta b_{s n}
\end{array}\right]^{T},(s=1,2) .
$$

Now, by inserting relations Eq.(5) and Eq.(6) into Eq.(3) and Eq.(4) and applying the Galerkin procedure to eliminate the time parameter $\tau$ through the orthogonality conditions yields

$$
<g(\tau), f(\tau)>=\frac{1}{2 \pi} \int_{0}^{2 \pi} f(\tau) g(\tau) d \tau=0,
$$

where by neglecting the higher order terms we can obtain the following system of algebraic equations as

$$
\left[\begin{array}{ll}
\mathbf{K}_{11} & \mathbf{K}_{12} \\
\mathbf{K}_{21} & \mathbf{K}_{22}
\end{array}\right]\left\{\begin{array}{l}
\Delta \mathbf{A}_{1} \\
\Delta \mathbf{A}_{2}
\end{array}\right\}=\left\{\begin{array}{l}
\mathbf{R}_{1} \\
\mathbf{R}_{2}
\end{array}\right\}+\left\{\begin{array}{l}
\mathbf{V}_{1} \\
\mathbf{V}_{2}
\end{array}\right\} \Delta \Omega
$$

where $\mathbf{K}_{i j}, \mathbf{R}_{i j}$ and $\mathbf{V}_{i j}$ are the matrices and $\mathbf{F}_{i}$ are the vectors defined as

$$
\begin{gathered}
\mathbf{K}_{11}=\int_{0}^{2 \pi}\left\{\Omega_{0}^{2} \mathbf{C}^{T} \ddot{\mathbf{C}}+\Omega_{0} \delta \mathbf{C}^{T} \dot{\mathbf{C}}+\left(\omega_{0}^{2}+2 \xi x_{0}+3 \gamma x_{0}^{2}+h p(\tau)\right) \mathbf{C}^{T} \mathbf{C}\right\} d \tau \\
\mathbf{K}_{12}=-\int_{0}^{2 \pi}\left\{\chi \mathbf{C}^{T} \mathbf{C}\right\} d \tau, \quad \mathbf{K}_{21}=\int_{0}^{2 \pi}\left\{k \Omega_{0} \mathbf{C}^{T} \dot{\mathbf{C}}\right\} d \tau \\
\mathbf{K}_{22}=\int_{0}^{2 \pi}\left\{\Omega_{0} \mathbf{C}^{T} \dot{\mathbf{C}}+\lambda \mathbf{C}^{T} \mathbf{C}\right\} d \tau \\
\mathbf{R}_{1}=-\int_{0}^{2 \pi}\left\{\Omega_{0}^{2} \mathbf{C}^{T} \ddot{\mathbf{C}}+\Omega_{0} \delta \mathbf{C}^{T} \dot{\mathbf{C}}+\left(\omega_{0}^{2}+\xi x_{0}+\gamma x_{0}^{2}+h p(\tau)\right) \mathbf{C}^{T} \mathbf{C}\right\} d \tau \mathbf{A}_{1}+
\end{gathered}
$$




$$
\begin{gathered}
+\int_{0}^{2 \pi}\left\{\chi \mathbf{C}^{T} \mathbf{C}\right\} d \tau \mathbf{A}_{2}+f \int_{0}^{2 \pi}\left\{\mathbf{C}^{T} \cos (\tau)\right\} d \tau \\
\mathbf{R}_{2}=-\int_{0}^{2 \pi}\left\{k \Omega_{0} \mathbf{C}^{T} \dot{\mathbf{C}}\right\} d \tau \mathbf{A}_{1}-\int_{0}^{2 \pi}\left\{\Omega_{0} \mathbf{C}^{T} \dot{\mathbf{C}}+\lambda \mathbf{C}^{T} \mathbf{C}\right\} d \tau \mathbf{A}_{2} \\
\mathbf{V}_{1}=-\int_{0}^{2 \pi}\left\{2 \Omega_{0} \mathbf{C}^{T} \ddot{\mathbf{C}}+\delta \mathbf{C}^{T} \dot{\mathbf{C}}\right\} d \tau \mathbf{A}_{1} \\
\mathbf{V}_{2}=-\int_{0}^{2 \pi}\left\{k \mathbf{C}^{T} \dot{\mathbf{C}}\right\} d \tau \mathbf{A}_{1}-\int_{0}^{2 \pi}\left\{\mathbf{C}^{T} \dot{\mathbf{C}}\right\} d \tau \mathbf{A}_{2} .
\end{gathered}
$$

Eq.(9) can be written in a simplified form as

$$
\mathbf{K} \Delta \mathbf{A}=\mathbf{R}+\mathbf{V} \Delta \Omega
$$

To determine one periodic solution for a specific value of the frequency parameter, we set $\Delta \Omega=0$ into the system of linear algebraic equations (Eq.(11)). Then, by using the Newton-Raphson iterative procedure, where solution process starts with the initialization of the coefficients $\mathbf{A}$ in such a manner that the tangent stiffness matrix $[\mathbf{K}]$ is not a singular matrix. By using the iterative procedure we can find the solution of $\Delta \mathbf{A}$ iteratively by solving the following equations

$$
\Delta \mathbf{A}=[\mathbf{K}]^{-1} \mathbf{R} \quad \longrightarrow \quad \mathbf{A}_{i+1}=\mathbf{A}_{i}+\Delta \mathbf{A}_{i+1},
$$

where iterations are performed until the residue Euclidean norm $\|\mathbf{R}\|$ is smaller than a preset tolerance $10^{-12}$, such that the corrective vector term $\mathbf{R}$ tends to zero when the values of coordinates tend to exact solutions. The next periodic solution can be determined by adding the small value $\Delta \Omega$ to the frequency parameter and using the Newton-Raphson iterative procedure, where the previous frequency step is used as the initial guess to obtain the solution for the current frequency. This type of the path-following method i.e. the continuation method is known as the "natural parameter continuation". However, using this type of the path-following method for tracing the frequency response, the periodic solution can be found in the vicinity of some bifurcation point (turning point), for which the tangent stiffens matrix $\mathbf{K}$ becomes singular. In order to eliminate failures in the frequency response due to bifurcation points, the numerical path-following method is employed in a manner similar to [48, 49]. In general, this method is based on the predictorcorrector procedures that trace the periodic solution branches i.e. amplitude-frequency response curves of the proposed nonlinear system. By using the continuation method in combination with the Floquet stability theory, the stable and unstable branches of periodic responses, as well as bifurcation points, are determined by using the methodology described in the following subsections.

\subsection{The path-following method}

The initialization of the continuation process begins with the determination of the periodic responses from an arbitrary initial state, mostly distant from the resonant state, where the response amplitudes are small. After finding the initial periodic solutions of the nonlinear system, the predictor-corrector procedure is used to perform point-to-point calculation to obtain the corresponding branches of frequency responses as shown in [48-50]. The pseudo-arc-length is one of the most used parametrisation approaches in the path-following, which allows one to trace the solutions across limit points and loops. By introducing the parameter $\eta$, the augmented equation is given as

$$
g(\mathbf{X})-\eta=0,
$$

where $\mathbf{X}=[\mathbf{A}, \Omega]^{T}$ and $g(\mathbf{X})=\mathbf{X}^{T} \mathbf{X}$. The slope is defined in terms of the two previous points $\mathbf{X}_{k-1}$ and $\mathbf{X}_{k-2}$ on the response curves, which is given as

$$
\mathbf{X}^{\prime}=\frac{\mathbf{X}_{k-1}-\mathbf{X}_{k-2}}{\left\|\mathbf{X}_{k-1}-\mathbf{X}_{k-2}\right\|}
$$

where the first predicted solution is calculated based on the first two periodic solutions obtained from IHBM

$$
\mathbf{X}_{u}=\mathbf{X}_{k-1}+\Delta \eta \mathbf{X}^{\prime}
$$

To obtain the periodic solution, one need to extend the tangent stiffness matrix by introducing the augmented equation and then applying the Newton-Raphson iterative procedure for the corrected solution. 
The augmented equation is based on Taylor's expansion from Eq.(13). Further, by extending Eq.(11) with augmented equation, the tangent stiffness matrix and residual vector can be written as

$$
\left[\begin{array}{cc}
\mathbf{K} & \mathbf{V} \\
\frac{\partial g}{\partial \mathbf{A}} & \frac{\partial g}{\partial \Omega}
\end{array}\right]\left\{\begin{array}{c}
\Delta \mathbf{A} \\
\Delta \Omega
\end{array}\right\}=\left\{\begin{array}{c}
\mathbf{R} \\
\Delta \eta-g
\end{array}\right\} .
$$

The initialization of the path-following method is starting from the relation given in Eq.(11), where the two periodic solutions are determined. Then, by using the extended Jacobian matrix with augmented equation and the predictor-corrector methodology tracing of the frequency response curves becomes a trivial task. The parameter $\Delta \eta$ is adopted as the arc increment having a small value. For higher accuracy of periodic solutions the introduction of the value of tolerance can vary in the range from $10^{-10}$ to $10^{-12}$. Setting small tolerances leads to tracing of the amplitude-frequency curves without breaks. For more details please refer to the following literature $[49,50]$.

\section{Stability analysis}

Once the periodic solution is determined in the form of Fourier series Eq.(6) for a particular value of $\Omega$, we can investigate the local stability of such a solution by using the method based on the Floquet theory $[47,48]$. Here, the methodology based on the Floquet theory developed in [51] is used for the approximation of the transition matrix during one period. The local stability analysis starting from the linearised equations Eq.(3) and Eq.(4) can be expressed in a general form as

$$
\mathbf{W}(\ddot{\mathbf{y}}, \dot{\mathbf{y}}, \mathbf{y}, \Omega, \tau)=\mathbf{0},
$$

where

$$
\mathbf{y}=\left[y_{1}(\tau), y_{2}(\tau), \ldots y_{N}(\tau)\right],
$$

is the $N$ - dimensional displacement vector. Inserting small perturbations $\Delta \mathbf{y}(\tau)$ in a neighbourhood of the previously determined periodic solution $\mathbf{y}_{0}(\tau)$ as follows

$$
\mathbf{y}=\mathbf{y}_{0}+\Delta \mathbf{y}(\tau)
$$

the local stability of periodic solutions can be analysed like the system with variable coefficients in terms of small perturbations $\Delta \mathbf{y}(\tau)$. By inserting Eq.(18) into the system of equations Eq.(17) and after linearisation one can obtain the system of linear differential equations with time-periodic coefficients as

$$
\left(\frac{\partial \mathbf{W}}{\partial \ddot{\mathbf{y}}}\right)_{0} \Delta \ddot{\mathbf{y}}(\tau)+\left(\frac{\partial \mathbf{W}}{\partial \dot{\mathbf{y}}}\right)_{0} \Delta \dot{\mathbf{y}}(\tau)+\left(\frac{\partial \mathbf{W}}{\partial \mathbf{y}}\right)_{0} \Delta \mathbf{y}(\tau)=\mathbf{0}
$$

in which $\mathbf{y}_{0}(\tau)=[x(\tau), v(\tau)]^{T}$ are the previously obtained periodic solutions. To determine the stability of the obtained periodic solutions by using the multi-variable Floquet theory, the transformation of Eq.(19) into the state-space form should be performed as

$$
\frac{d \mathbf{Y}}{d \tau}=\mathbf{P}(\tau) \mathbf{Y}
$$

where $\mathbf{Y}(\tau)=[\Delta \mathbf{y}, \Delta \dot{\mathbf{y}}]^{T}$ and $\mathbf{P}(\tau)$ denotes the periodic matrix with the period $T$. The stability criteria based on the Floquet theory is used for determination of the local stability of periodic solutions and it is related to determination of the Floquet multipliers as given in [51]. The Floquet multipliers are represented by the eigenvalues of the monodromy matrix. By solving the corresponding eigenvalue problem, for the case when all Floquet multipliers are located inside the unit circle centred at the origin of the complex plane, determined periodic solutions are stable or asymptotically stable. Otherwise, when the values of Floquet multipliers are outside of the unit circle, the periodic solutions are unstable [47]. Depending on where the Floquet multipliers or a pair of complex conjugate multipliers crosses the complex plane unit circle, different bifurcation points can be detected.

Introduction of the Hsu procedures for numerical approximation of the transition matrix during one period leads to numerical determination of Floquet multipliers as a solution of the corresponding eigenvalue problem [47]. It is assumed that the period $T=2 \pi$ of the periodic solution $y_{0}(\tau)$ is divided into $N_{k}$ subintervals, where the $k$-th interval is equal to $\Delta_{k}=\tau_{k}-\tau_{k-1}$ for $\tau_{k}=k T / N_{k}$. Furthermore, $\mathbf{P}(\tau)$ is the 
continuous periodic matrix with respect to $\tau$ and period $T$, where in the $k$-th interval it can be replaced by the constant matrix provided in the case when $N_{k}$ is chosen to be sufficiently large, where

$$
\mathbf{P}_{k}=\frac{1}{\Delta_{k}} \int_{\tau_{k-1}}^{\tau_{k}} \mathbf{P}(\tau) d \tau
$$

The monodromy (transition) matrix can be written in the following form

$$
\mathbf{M}=\prod_{i=1}^{N_{k}} e^{\mathbf{P}_{i} \Delta_{i}}=\prod_{i=1}^{N_{k}}\left(\mathbf{I}+\sum_{j=1}^{N_{j}} \frac{\left(\mathbf{P}_{i} \Delta_{i}\right)^{j}}{j !}\right),
$$

where $N_{j}$ denotes the number of terms in Taylor series for approximation of the constant matrix $\mathbf{P}_{k}$. From the monodromy matrix $\mathbf{M}$ one can obtain Floquet multipliers as eigenvalues of Eq.(22) in the form

$$
\operatorname{det}(\mathbf{M}-\sigma \mathbf{I})=0 .
$$

For the system given in Eq.(3) and Eq.(4), the periodic matrix $\mathbf{P}(\tau)$ can be obtained in the following form

$$
\mathbf{P}(\tau)=\left[\begin{array}{ccc}
0 & 1 & 0 \\
H(\tau) & -\frac{\delta}{\Omega} & \frac{\chi}{\Omega^{2}} \\
0 & -k & -\frac{\lambda}{\Omega}
\end{array}\right]
$$

where

$$
H(\tau)=-\frac{1}{\Omega^{2}}\left(\omega_{0}^{2}+2 \xi x_{0}+3 x_{0}^{2} \gamma+h p(\tau)\right) .
$$

In this study, for approximation of the monodromy matrix the next values of $N_{k}=5000$ and $N_{j}=5$ are adopted.

\section{Numerical results}

The combination of the IHB and the path-following methods are introduced to trace the branches of periodic solutions of the nonlinear model of parametrically amplified piezoelectric EH with direct harmonic excitation. The obtained diagrams are showing periodic responses given in the form of amplitude-frequency curves. It is demonstrated that the parametric amplification phenomena has a significant influence on the response amplitudes for both types of parametric excitation $p(\tau)=h \sin (\tau)$ and $p(\tau)=h \cos (2 \tau)$. The external harmonic excitation is introduced in the following form $f \cos (\tau)$. To understand the effect of the parametric amplification on harmonically excited system, periodic response curves computed with and without the influence of parametric amplification are compared. Moreover, the results obtained by IHBM are verified with the results they from direct numerical integration (NI). The second part of the numerical results section is devoted to the analysis of the influence of quadratic and cubic nonlinearities as well as the magnitude and type of parametric excitation on the EH response. The results revealed the importance of the first three harmonics on the dynamic behaviour of the nonlinear EH model. Therefore, the upper, lower, and unstable branches of periodic response for the first three harmonics are observed in the numerical study. Furthermore, different nonlinear phenomena such as multiple jumps, loops, and a super-harmonic resonant peak are also identified.

The response amplitudes are defined based on the Fourier series Eq.(6) in the following form

$$
\begin{aligned}
& x_{0}(\tau)=A_{10}+A_{11} \cos \left(\tau+\phi_{11}\right)+A_{12} \cos \left(2 \tau+\phi_{12}\right)+\ldots \\
& v_{0}(\tau)=A_{20}+A_{21} \cos \left(\tau+\phi_{21}\right)+A_{22} \cos \left(2 \tau+\phi_{22}\right)+\ldots
\end{aligned}
$$

where

$$
A_{j 0}=a_{j 0}, \quad A_{j k}=\sqrt{a_{i j}^{2}+b_{j k}^{2}}, \quad \phi_{j k}=\tan ^{-1}\left(b_{j k} / a_{i j}\right), \quad(j=1,2 ; \quad k=1,2 \ldots, 8) .
$$




\subsection{Verification}

To demonstrate the accuracy of the proposed approach in determination of the periodic solutions and corresponding frequency-response diagrams, the obtained results from IHBM and continuation technique are verified with the results from direct numerical integration. Moreover, results obtained with and without the influence of parametric excitation are presented to highlight its effect on the harmonically excited EH system. The following dimensionless parameters are adopted: $\delta=0.025, \quad \omega_{0}=0.5, \quad \xi=0.7, \quad \gamma=$ $1, \quad \chi=0.5, \quad f=0.01, \quad \lambda=0.07, \quad k=0.5$. Two values of the parametric excitation are used $h=0.1$ and $h=0$. Moreover, the number of adopted harmonics in the Fourier series is $M=8$. In Fig.2 and Fig. 3 the frequency response curves are given for amplitudes $A_{11}$ and $A_{21}$ corresponding to both types of parametric excitation, $p(\tau)=h \sin (\tau)$ and $p(\tau)=h \cos (2 \tau)$. The response amplitudes $A_{11}$ and $A_{21}$ corresponding to the mechanical displacement and electrical voltage, respectively are given on the ordinate axis while excitation frequency $\Omega$ is on the abscissa, (Fig.2 a) and Fig.3 a)). Two periodic orbits are selected from the response curve $A_{11}$, and validated with the direct numerical integration. The periodic solutions are depicted in the phase plane, where the velocity is given on the ordinate axis while the displacement is on the abscissa, as shown in sub-figures b) and c) of Fig.2 and Fig.3.

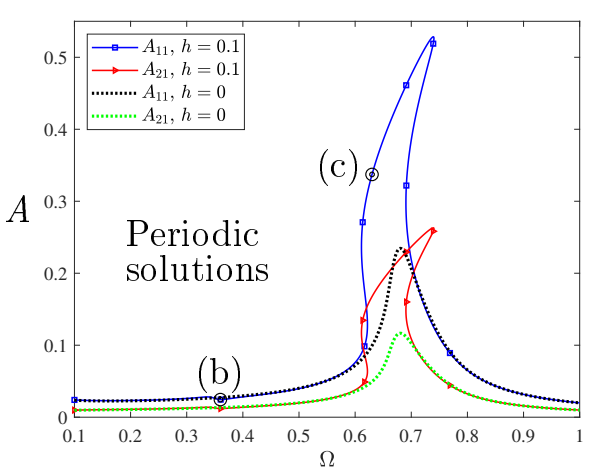

(a)

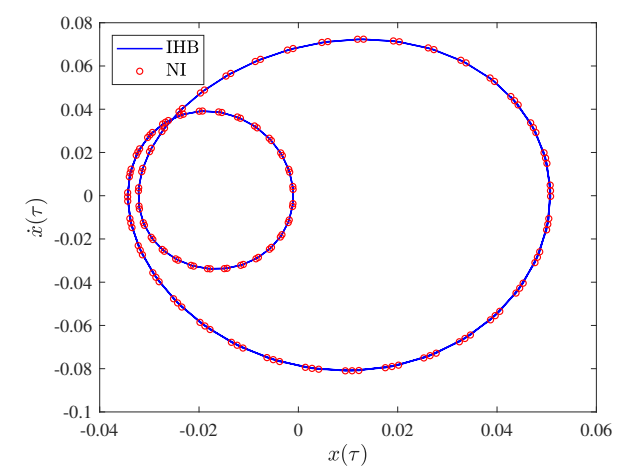

(b)

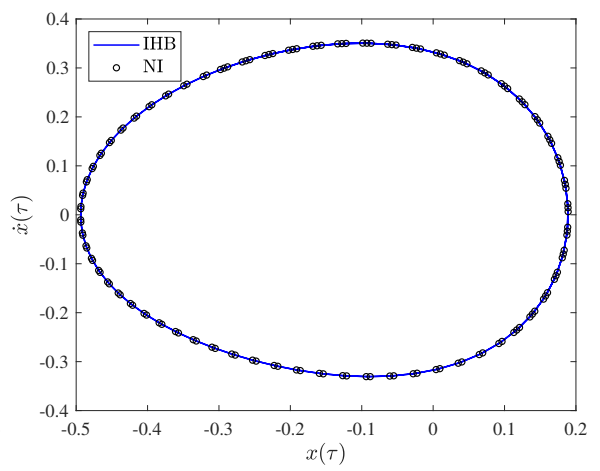

(c)

Figure 2: The amplitude-frequency response curves $A_{11}$ and $A_{21}$ of the nonlinear energy harvester with combined parametric $(p(\tau)=h \sin (\tau))$ and external excitation for two values of the parametric excitation magnitude $h=0-$ dotted line and $h=0.1$ - solid line are given in the sub-figure (a). The periodic responses obtained by the incremental harmonic balance (IHB) method and direct numerical integration (NI) taken far away from the resonant state is given in the sub-figure (b), and close to resonant state in the sub-figure (c).

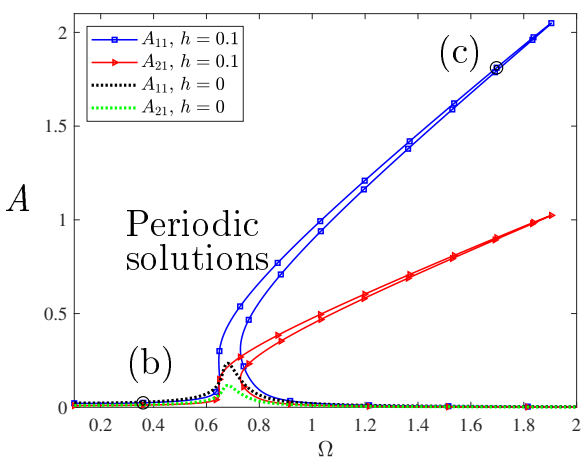

(a)

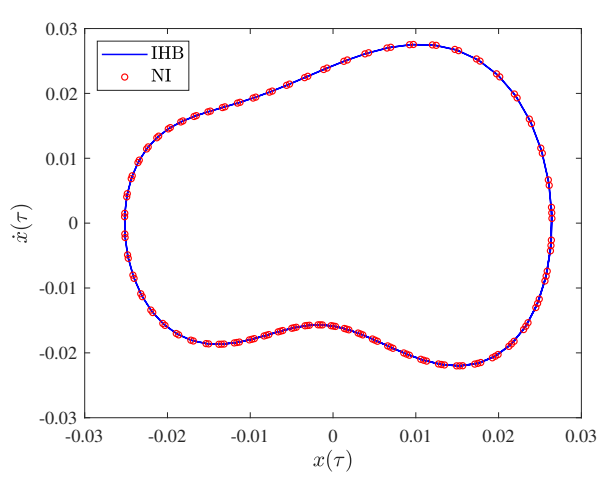

(b)

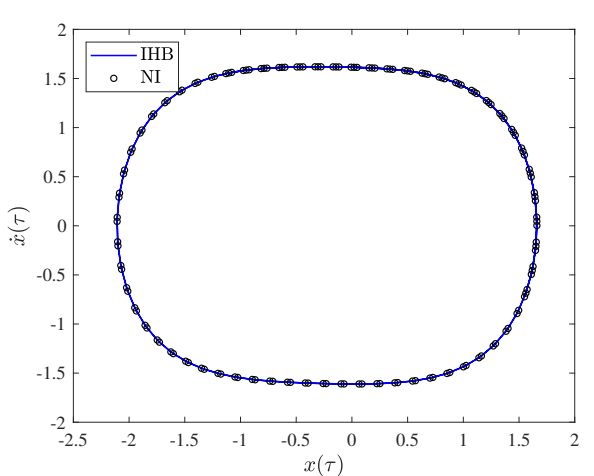

(c)

Figure 3: The amplitude-frequency response curves $A_{11}$ and $A_{21}$ of the nonlinear energy harvester with combined parametric $(p(\tau)=h \cos (2 \tau))$ and external excitation for two values of the parametric excitation magnitude $h=0-$ dotted line and $h=0.1$ - solid line are given in sub-figure (a). The periodic response obtained by the incremental harmonic balance (IHB) method and direct numerical integration (NI) taken far away from the resonant state is given in the sub-figure (b), and close to resonant state in the sub-figure (c).

Fig.2 a) shows the influence of the parametric excitation magnitude $h$ on the amplitude-response curves, where excitation frequency $\Omega$ varies in the range $0.1-1$. It can be observed that the response amplitudes $A_{11}$ and $A_{21}$ are almost doubled for the parametric excitation magnitude equal to $h=0.1 \mathrm{in}$ comparison to the case when $h=0$. This implies that the introduced parametric excitation leads to parametric amplification 
of the harmonic response, which was the main aim of introducing the parametric amplification. Moreover, both response amplitudes $A_{11}$ and $A_{21}$ are exhibiting the nonlinear behaviour in the form of softening and hardening spring nonlinearity with jump effects. More details on forward and backward frequency sweeping are provided in the following section based on determined bifurcation points and unstable periodic solutions. To verify the periodic solutions obtained by the IHBM we select two points on the response curve $A_{11}$, and show them in the phase plane against the periodic solutions obtained by the numerical integration method applied to Eq.(3) and Eq.(4) (sub-figures b) and c) in Fig.2). One can observe an excellent matching of curves obtained by two different methods.

Fig. 3 a) shows response amplitudes $A_{11}$ and $A_{21}$ obtained for the parametric excitation in the form $p(\tau)=$ $h \cos (2 \tau)$. The same values of dimensionless parameters as in the previous case of nonlinear piezoelectric $\mathrm{EH}$ are adopted. Similar to the previous parametric excitation case one can also observe an increase of the response amplitudes $A_{11}$ and $A_{21}$ and nonlinear effects in the system. As expected, the amplification phenomena caused by $p(\tau)=h \cos (2 \tau)$ in combination with cubic nonlinearity has a dominant effect in the response amplitude. In this case, the frequencies are tuned in the form of two-to-one resonance between parametric and external excitation causing the combined resonant state. Furthermore, it can be noticed that the frequency response curves are displaying the hardening spring nonlinearity, which is more prominent than the softening spring nonlinearity. From the physical viewpoint, the cosine type of parametric excitation in combination with the external excitation causes a significant amplification of the EH voltage for a wide spectrum of excitation frequencies. Fig. 3 b) and c) shows two periodic solutions chosen from the response amplitudes in Fig.3 a) and obtained by both IHBM and direct NI method, where fine agreement of the results is achieved.

In summary of the above results, it can be stated that the parametric amplification phenomena plays important role in an increase of the response amplitudes of externally excited nonlinear piezoelectric EH, which then leads to higher harvesting voltage and corresponding power. Moreover, one can observe that the system subjected to cosine parametric excitation amplifies the response amplitude more than the sinusoidal form of parametric excitation. Finally, the periodic solutions of the mechanical and electrical responses of the nonlinear EH are verified with the numerical results from the Runge-Kutta (RK) method obtained by using the Matlab built-in function 'ode45' and excellent agreement of the results is demonstrated.

\subsection{The effects of cubic nonlinearity}

In this section, an investigation of the effects of cubic type nonlinearity $\gamma$ on the frequency response curves in terms of the first three amplitudes $\left(A_{11}, A_{21}, A_{12}, A_{22}\right.$, and $\left.A_{13}, A_{23}\right)$ is performed. The response amplitudes $A_{1 j}$ and $A_{2 j}$ for $j=1,2,3$, are defined in Eq.(25). To study the effects of cubic nonlinearity on the parametric amplification of the response amplitudes, two sets of system parameters are used, where the parametric excitation is adopted as $p(\tau)=h \sin (\tau)$ and $p(\tau)=h \cos (2 \tau)$, and the values of cubic nonlinearity as $\gamma=0.5$ and $\gamma=3$. Other system parameters are: $\delta=0.025, \quad \omega_{0}=0.5, \quad \xi=0.5, \quad h=$ $0.2, \quad \chi=0.5, \quad f=0.01, \quad \lambda=0.07, \quad k=0.5$. Fig.4 - Fig.7 shows the frequency response amplitudes $\left(A_{11}, A_{12}, A_{A 13}\right)$ of the mechanical displacement (blue solid line) and response amplitudes $\left(A_{21}, A_{22}, A_{A 23}\right)$ of the electrical voltage (black dashed line) for the proposed EH device. The effects of different types of parametric excitation for higher and lower values of the cubic nonlinearity are also studied. A significant difference in the response amplitudes of sinusoidal and cosine form of the parametric excitation can be observed, especially in the vicinity of the resonance frequency. From these response curves, a complex behaviour characterized by different nonlinear phenomena such as mixed softening and hardening spring nonlinearity or multiple jump-up jump-down phenomena can be observed.

Fig.4 shows the parametrically amplified response of directly excited EH device when the parametric excitation is adopted in the form $p(\tau)=h \sin (\tau)$. The first three trsed amplitudes corresponding to the first three modes of Fourier series solution are having both stable and unstable branches of the response. The response amplitudes $A_{11}$ and $A_{21}$ (Fig.4 a)) formed by forward and backward frequency sweeping are analysed in detail. Similar frequency sweeping can be used to trace the response amplitudes of higher modes $\left(A_{12}, A_{22}\right.$ and $A_{13}, A_{23}$ ). One can notice that the response amplitude $A_{11}$ (or $A_{21}$ ) linearly increases for an increase of $\Omega$ until the moment when the periodic response loses its stability and jump-up phenomena appears i.e. the response amplitude suddenly jumps to some higher values. The instability point at which the periodic solution loses its stability is known as the saddle-node bifurcation. Further increase in the excitation frequency gives another branch of stable periodic solutions (known as the upper branch of periodic solutions) for which the amplitude grows until the value of excitation frequency $\Omega$ reaches a critical point representing the maximal value of the response amplitude (response peak), and where periodic solutions loses its stability 


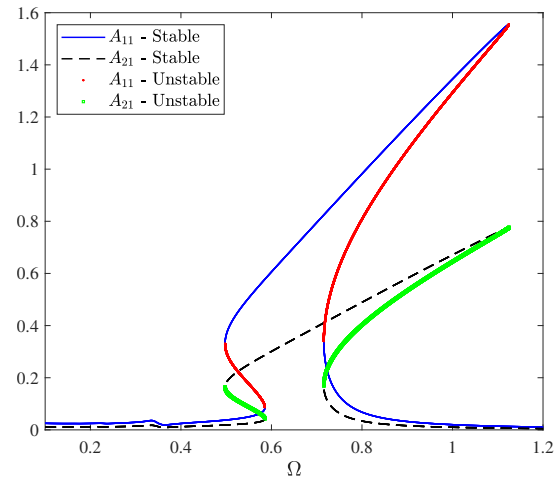

(a)

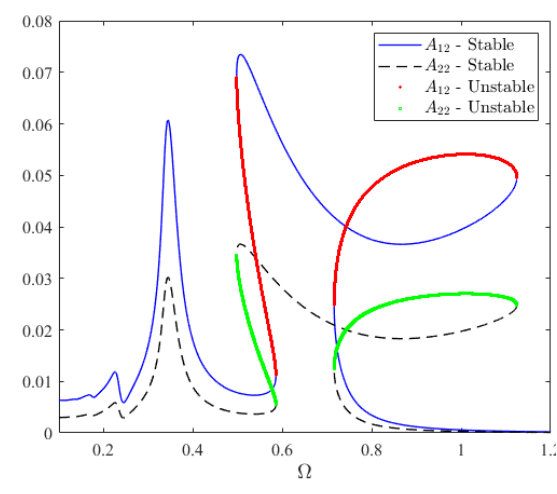

(b)

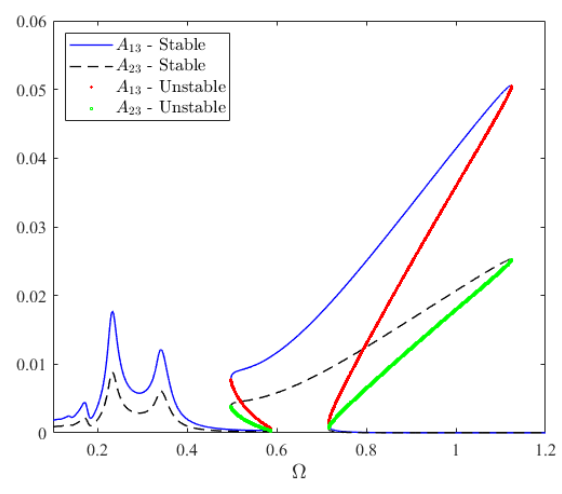

(c)

Figure 4: The amplitude-frequency response curves $A_{1 j}$ - solid line and $A_{2 j}$ - dashed line $(j=1,2,3)$ of the nonlinear energy harvester with parametric excitation $p(\tau)=h \sin (\tau)$ and the nonlinear cubic stiffness $\gamma=0.5$. The stable periodic solutions are represented by the blue solid line for $A_{11}$ and dashed back line for $A_{21}$ while unstable periodic solutions are represented by the red solid line for $A_{11}$ and green solid line for $A_{21}$. The following sub-figures shows (a) the amplitude-frequency response curves $A_{11}$ and $A_{21}$, (b) the amplitude-frequency response curves $A_{12}$ and $A_{22}$, (c) the amplitude-frequency response curves $A_{13}$ and $A_{23}$.

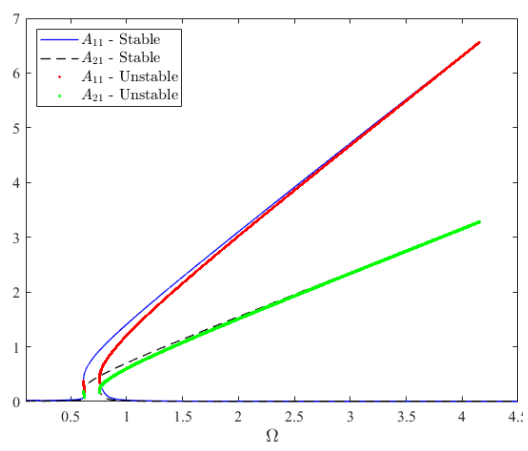

(a)

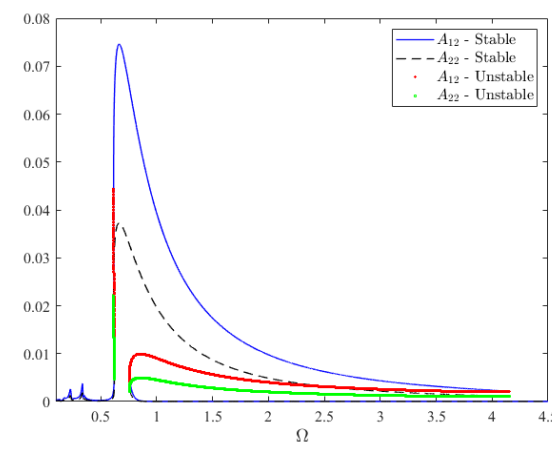

(b)

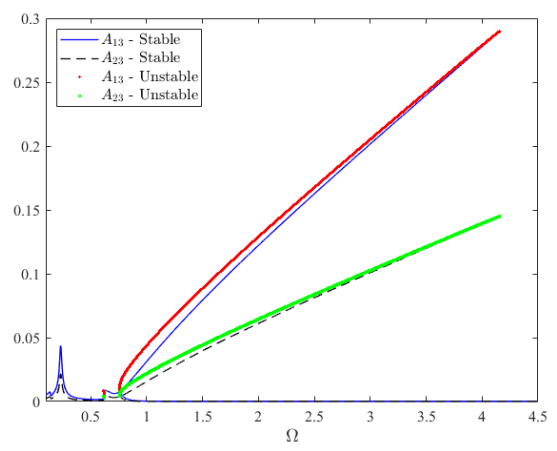

(c)

Figure 5: The amplitude-frequency response curves $A_{1 j}$ - solid line and $A_{2 j}$ - dashed line $(j=1,2,3)$ of the nonlinear energy harvester with parametric excitation $\gamma=0.5$. The stable periodic solutions are represented by the blue solid line for $A_{11}$ and dashed black line for $A_{21}$ while unstable periodic solutions are represented by the red solid line for $A_{11}$ and green solid line for $A_{21}$. The following sub-figures shows (a) the amplitude-frequency response curves $A_{11}$ and $A_{21}$, (b) the amplitude-frequency response curves $A_{12}$ and $A_{22}$, (c) the amplitude-frequency response curves $A_{13}$ and $A_{23}$.

again. This instability point implies the jump-down phenomena of the response amplitude i.e. a sudden jump of the response amplitude to some lower values. This process of increasing the excitation frequency from some small initial values towards higher values, passing through the resonance state and than continuing to certain finite and higher values of the excitation frequency is known as forward frequency sweeping. In the backward frequency sweeping, the starting point of excitation frequency $\Omega$ is taken to be higher than the resonant frequency. By moving backward, small increases in the response amplitude can be observed until the critical point where the periodic solution loses its stability, which leads to the appearance of jump-up phenomena. Further decrease of the excitation frequency is followed by the appearance of the stable (upper) branch of periodic responses. However, after some critical point the periodic solutions become unstable again, which is indicated by the appearance of jump-down phenomena. The branches of unstable periodic solutions can be de detected between two instability points and they are represented by the red and green solid lines. It can be observed that the mixed quadratic and cubic nonlinearities in the proposed EH system are causing the appearance of multiple jump-up and jump-down phenomena in response amplitudes, which leads to response amplitudes consisting of both stable and unstable branches of periodic solutions. Moreover, for the response amplitudes $A_{12}, A_{22}, A_{13}$ and $A_{23}$ even more complex dynamic behaviour can be observed as shown in sub-figures b) and c) of Fig.4.

Fig.5 shows the frequency response curves when the system is amplified by parametric excitation $p(\tau)=$ $h \cos (2 \tau)$. As expected, an increase of the excitation frequency $\Omega$ leads to an increase of the response amplitudes $A_{11}$ and $A_{21}$, especially near the resonant frequency, where periodic solution loses its stability due to the saddle-node bifurcation Fig.5. Moreover, the response amplitudes are exhibiting the mixed softening- 
hardening spring nonlinearity, where the softening effect is more prominent when the system is excited by the parametric term $p(\tau)=h \sin (\tau)$ as shown in Fig. 4 a). On the other hand, for the system excited by $p(\tau)=h \cos (2 \tau)$ the response amplitudes are for an order of magnitude larger (Fig.5 a) ) than those for the system excited by $p(\tau)=h \sin (\tau)$. Based on this observation, it can be concluded that the nonlinear EH model excited by $p(\tau)=h \cos (2 \tau)$ yields higher response amplitude $\left(A_{11}\right)$ and electrical voltage $\left(A_{21}\right)$ therefore becoming more relevant for the EH application. The multiple jumps can be detected (Fig.5) by forward and backward frequency sweeping caused by combining the quadratic and cubic type nonlinearities in the system. However, these multiple jumps can be also interpreted as regions with coexisting multiple periodic solutions, which can be easily detected at higher modes, sub-figures b) and c) in Fig.5.

The frequency response functions for higher harmonic amplitudes $\left(A_{12}, A_{22}, A_{13}\right.$ and $\left.A_{23}\right)$ are shown in Fig.4 b), c), Fig.5 b) and c). Two types of parametric excitation are considered. By performing forward and backward frequency sweeping, one can observe few resonant peaks with small response amplitudes before the excitation frequency reaches a value close to the resonance. In the vicinity of the resonant frequency, the response amplitude is dramatically increased. A qualitative change in response diagrams is caused by the combined effects of parametric excitation and mixed nonlinearities, which leads to the softeninghardening spring nonlinearity properties and multiple jump phenomena. By comparing the highest values of amplitudes for the second and third harmonics, it can be observed that the sinusoidal form of parametric excitation has the biggest effect on the second amplitude, much more than on other modes. However, as previously observed the cosine form of parametric excitation leads to higher values of the response amplitude and electrical voltage.

The frequency responses of the EH system are shown in Fig.4 and Fig. 5 for the value of cubic nonlinearity $\gamma=0.5$ and in Fig. 6 and Fig.7 for $\gamma=3$. The higher values of cubic nonlinearity increase the overall stiffness in the system and reduce the influence of parametric amplification on the harmonic signal. Furthermore, the maximum values of response amplitudes have been reduced by increasing the values of cubic nonlinearity, especially when the excitation frequency is close to the resonance. However, increasing the value of cubic nonlinearity can eliminate the effects of the quadratic one, which is particularly noticeable in the first mode as given in sub-figures (a). By cancelling the quadratic nonlinearity, the frequency response diagrams exhibit only hardening type nonlinearity, which leads to reduction of the unstable branches of periodic responses. It can be observed that only one unstable branch of periodic response is detected for every particular frequency response. This reduction in unstable branches causes the upper stable branch of periodic response to become narrower and therefore the range of operating frequency with higher response amplitude is reduced.

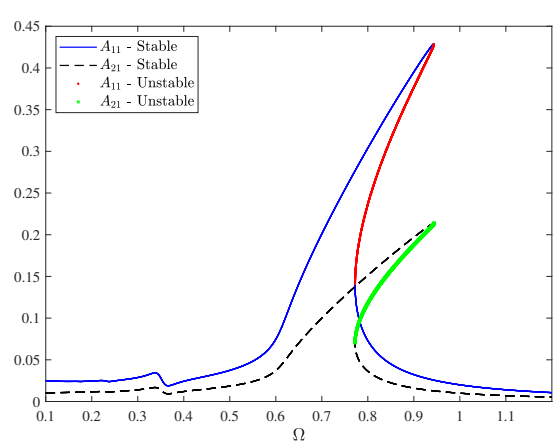

(a)

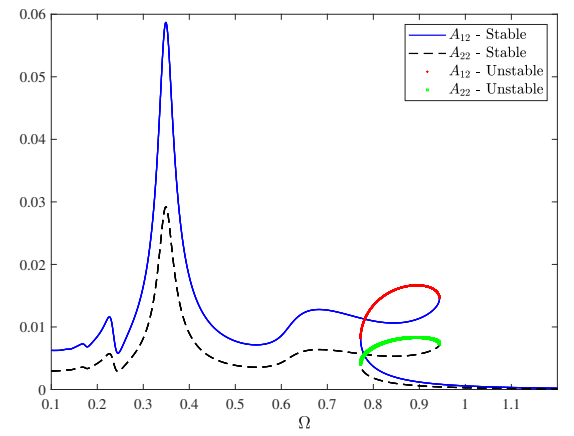

(b)

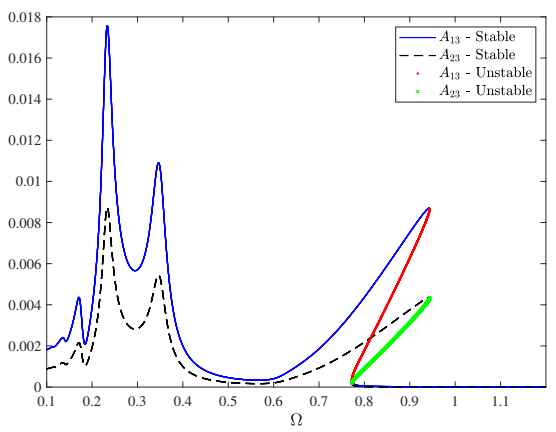

(c)

Figure 6: The amplitude-frequency response curves $A_{1 j}$ - solid line and $A_{2 j}$ - dashed line $(j=1,2,3)$ of the nonlinear energy harvester with the parametric excitation $p(\tau)=h \sin (\tau)$ and nonlinear cubic stiffness $\gamma=3$. The stable periodic solutions are represented by the blue solid line for $A_{11}$ and dashed back line for $A_{21}$ while unstable periodic solutions are represented by the red solid line for $A_{11}$ and green solid line for $A_{21}$. The following sub-figures shows (a) the amplitude-frequency response curves $A_{11}$ and $A_{21}$, (b) the amplitude-frequency response curves $A_{12}$ and $A_{22}$, (c) the amplitude-frequency response curves $A_{13}$ and $A_{23}$.

\subsection{The effect of the magnitude of parametric excitation}

In this subsection, the frequency response curves are determined to analyse the influence of the magnitude and type of parametric excitation on the nonlinear dynamic behaviour of the proposed EH. The parametric excitation is adopted in the sinusoidal and cosine form. The structural parameters adopted in this analysis are: $\gamma=1, \quad \delta=0.025, \quad \omega_{0}=0.5, \quad \xi=0.8, \quad \chi=0.5, \quad f=0.01, \quad \lambda=0.07, \quad k=0.5$. Fig.8 - Fig.11 depicts the amplitude-frequency response curves for $\left(A_{11}, A_{12}, A_{A 13}\right)$ (blue solid line) and $\left(A_{21}, A_{22}, A_{A 23}\right)$ 


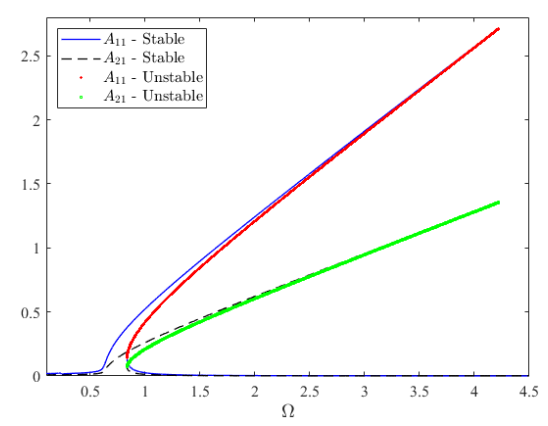

(a)

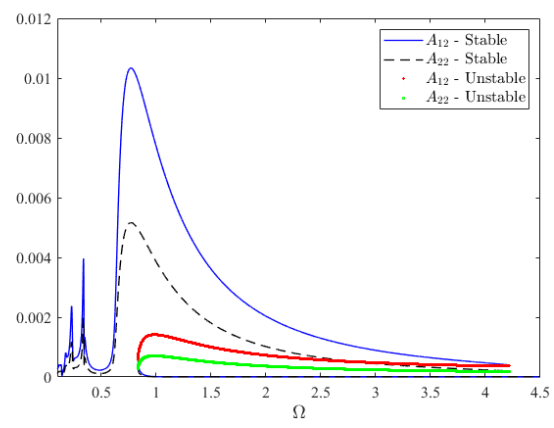

(b)

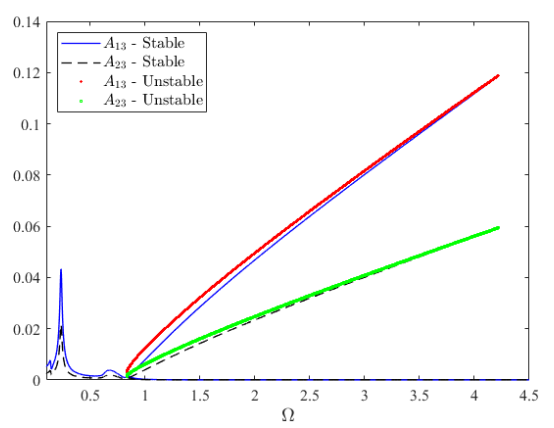

(c)

Figure 7: The amplitude-frequency response curves $A_{1 j}$ - solid line and $A_{2 j}$ - dashed line $(j=1,2,3)$ of the nonlinear energy harvester with the parametric excitation $p(\tau)=h \cos (2 \tau)$, where the nonlinear cubic stiffness is $\gamma=3$. The stable periodic solutions are represented by the blue solid line for $A_{11}$ and dashed back line for $A_{21}$ while the unstable periodic solutions are represented by the red solid line for $A_{11}$ and green solid line for $A_{21}$. The following sub-figures shows (a) the amplitude-frequency response curves $A_{11}$ and $A_{21}$, (b) the amplitude-frequency response curves $A_{12}$ and $A_{22}$, (c) the amplitude-frequency response curves $A_{13}$ and $A_{23}$.

(black dashed line). The most prominent influence of the parametric amplification phenomena can be detected by changing its magnitude. It can be observed that a light change in the magnitude of parametric excitation almost doubles the response amplitudes. This change is most noticeable for the first $\left(A_{11}\right.$ and the third $\left.A_{A 13}\right)$ harmonic amplitude, as shown in sub-figures a) and c) of Fig.8 - Fig.11. Moreover, nonlinear phenomena such as softening and hardening spring nonlinearity and jumps become more pronounced. By comparing the harmonic responses for lower and higher values of the magnitude of parametric excitation it can be noticed that an increase in its magnitude emphasizes the softening spring nonlinearity effect, especially for the sinusoidal type of excitation. Consequently, unstable branches of periodic response become also larger (red and green lines) while the upper stable branches become wider. Based on the previous results, it can be outlined that an increase in the magnitude of parametric excitation significantly increases the response amplitude of electrical voltage, which can improve the energy harvesting capabilities of the proposed EH device with PZT elements. This type of EH design has large potential for immense application in MEMS and NEMS devices.

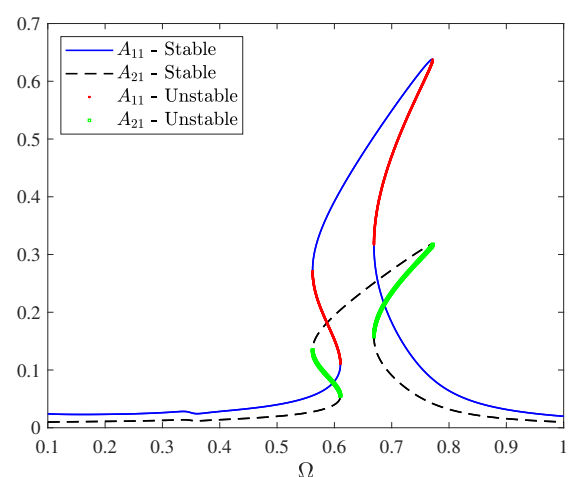

(a)

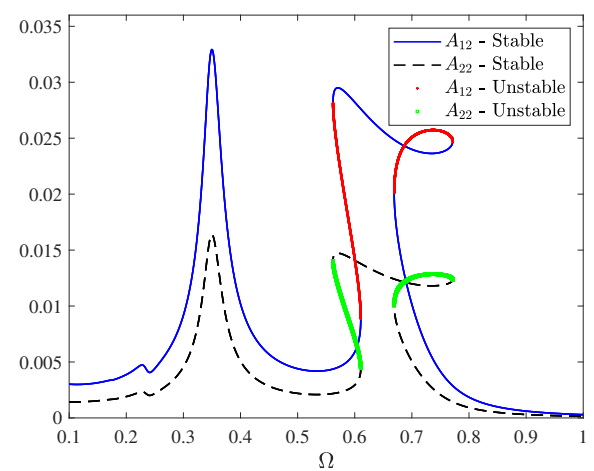

(b)

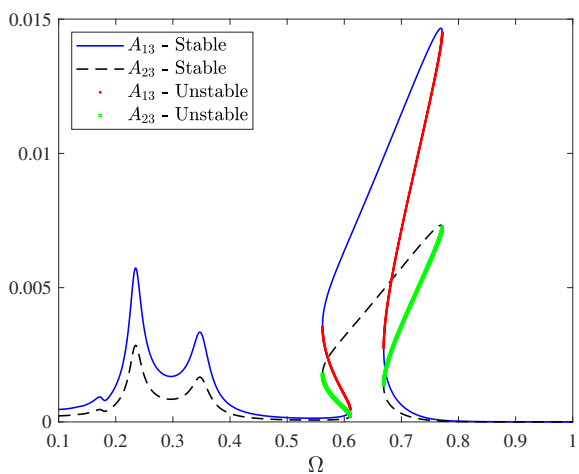

(c)

Figure 8: The amplitude-frequency response curves $A_{1 j}$ - solid line and $A_{2 j}$ - dashed line $(j=1,2,3)$ of the nonlinear energy harvester with parametric excitation $p(\tau)=h \sin (\tau)$ and $h=0.1$. The stable periodic solutions are represented by the blue solid line for $A_{11}$ and dashed black line for $A_{21}$ while unstable periodic solutions are represented by the red solid line for $A_{11}$ and green solid line for $A_{21}$. The following sub-figures shows (a) the amplitude-frequency response curves $A_{11}$ and $A_{21}$, (b) the amplitude-frequency response curves $A_{12}$ and $A_{22}$, (c) the amplitude-frequency response curves $A_{13}$ and $A_{23}$.

\subsection{The effects of quadratic nonlinearity and super-harmonic resonances}

In this section, the effect of quadratic nonlinearity $\xi$ on the frequency response curves is investigated for the harmonic amplitudes $\left(A_{11}\right.$ and $\left.A_{21}\right)$ of the nonlinear EH model. The parametric excitation $p(\tau)=$ $h \sin (\tau)$, values of quadratic nonlinearity $\xi=0.2 ; 0.4 ; 0.6$, and values of other system parameters $\delta=$ $0.025, \quad \omega_{0}=0.5, \quad \gamma=1, \quad h=0.3, \quad \chi=0.5, \quad f=0.01, \quad \lambda=0.07, \quad k=0.5$ are adopted in numerical simulations. Fig.12 shows the effect of change of quadratic nonlinearity term on frequency response curves for displacement amplitude $\left(A_{11}\right)$ - blue solid line and electrical voltage $\left(A_{21}\right)$ - black dashed line. 


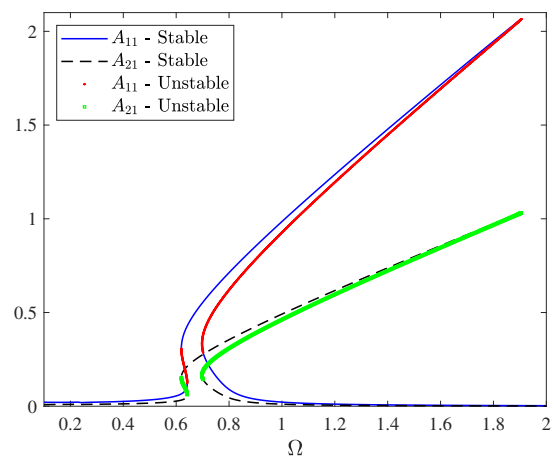

(a)

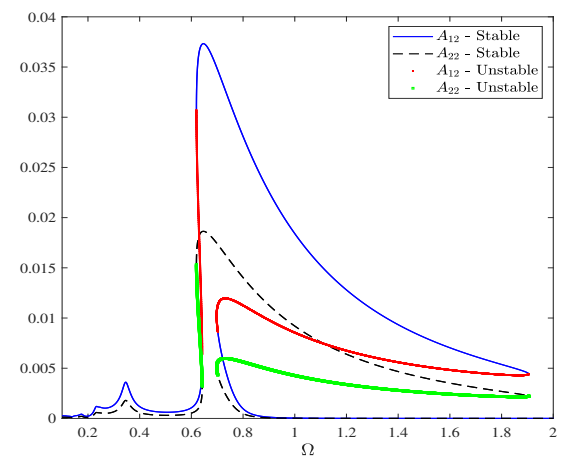

(b)

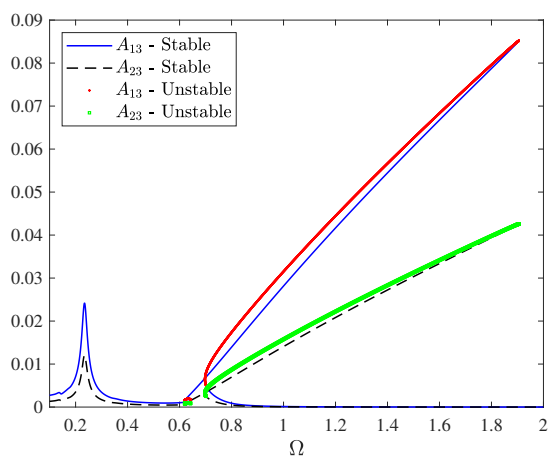

(c)

Figure 9: The amplitude-frequency response curves $A_{1 j}$ - solid line and $A_{2 j}$ - dashed line $(j=1,2,3)$ of the nonlinear energy harvester with parametric excitation $p(\tau)=h \cos (2 \tau)$ and $h=0.1$. The stable periodic solutions are represented by the blue solid line for $A_{11}$ and dashed back line for $A_{21}$ while unstable periodic solutions are represented by the red solid line for $A_{11}$ and green solid line for $A_{21}$. The following sub-figures shows (a) the amplitude-frequency response curves $A_{11}$ and $A_{21}$, (b) the amplitude-frequency response curves $A_{12}$ and $A_{22}$, (c) the amplitude-frequency response curves $A_{13}$ and $A_{23}$.

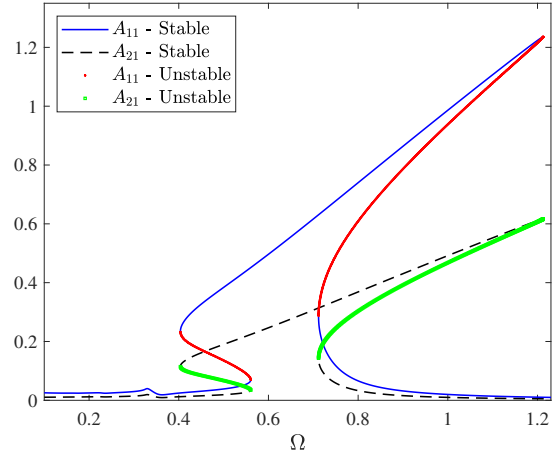

(a)

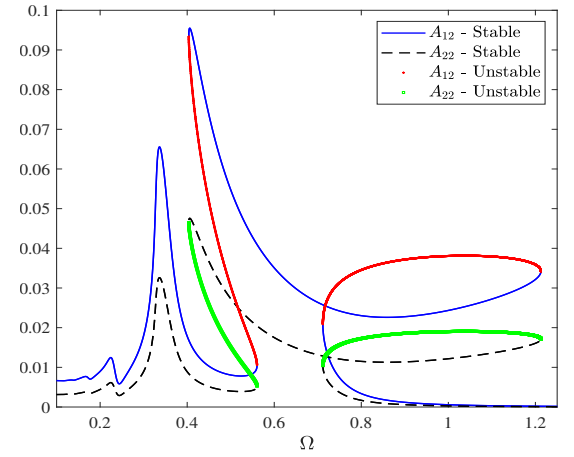

(b)

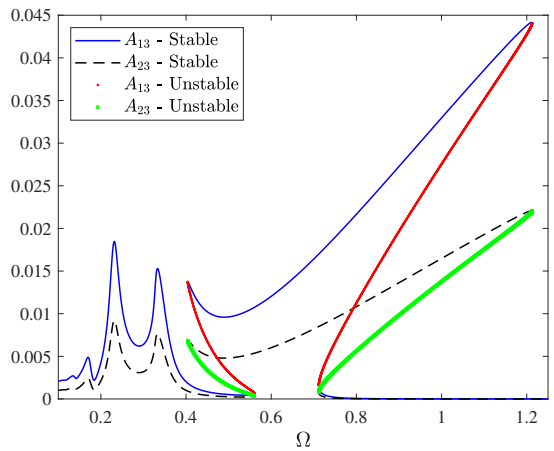

(c)

Figure 10: The amplitude-frequency response curves $A_{1 j}$ - solid line and $A_{2 j}$ - dashed line $(j=1,2,3)$ of the nonlinear energy harvester with parametric excitation $p(\tau)=h \sin (\tau)$ and $h=0.2$. The stable periodic solutions are represented by the blue solid line for $A_{11}$ and dashed back line for $A_{21}$ while unstable periodic solutions are represented by the red solid line for $A_{11}$ and green solid line for $A_{21}$. The following sub-figures shows (a) the amplitude-frequency response curves $A_{11}$ and $A_{21}$, (b) the amplitude-frequency response curves $A_{12}$ and $A_{22}$, (c) the amplitude-frequency response curves $A_{13}$ and $A_{23}$.

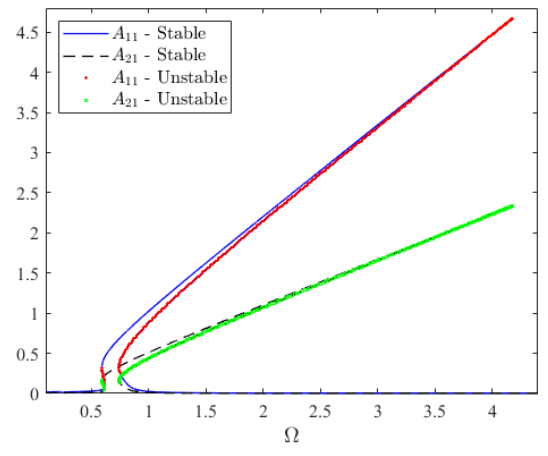

(a)

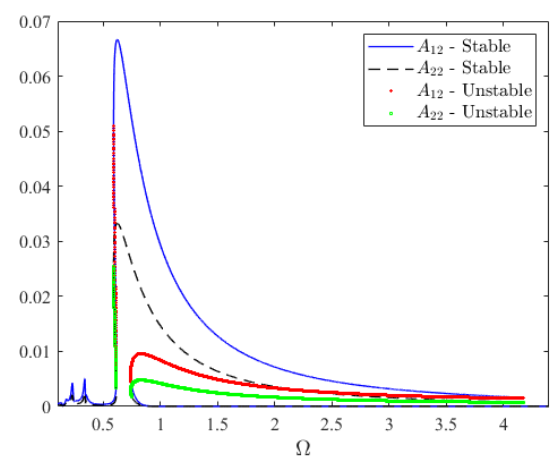

(b)

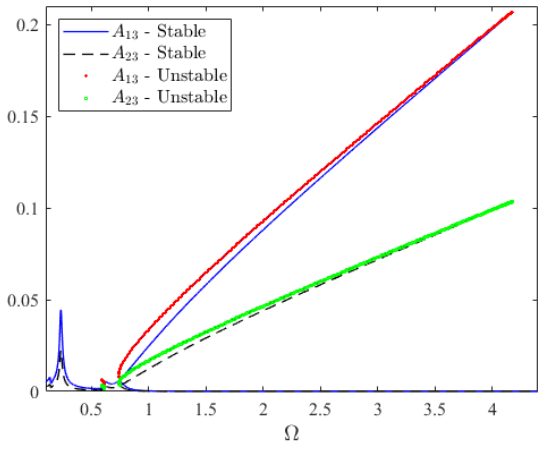

(c)

Figure 11: The amplitude-frequency response curves $A_{1 j}$ - solid line and $A_{2 j}$ - dashed line $(j=1,2,3)$ of the nonlinear energy harvester with parametric excitation $p(\tau)=h \cos (2 \tau)$ and $h=0.2$. The stable periodic solutions are represented by the blue solid line for $A_{11}$ and dashed back line for $A_{21}$ while unstable periodic solutions are represented by the red solid line for $A_{11}$ and green solid line for $A_{21}$. The following sub-figures shows (a) the amplitude-frequency response curves $A_{11}$ and $A_{21}$, (b) the amplitude-frequency response curves $A_{12}$ and $A_{22}$, (c) the amplitude-frequency response curves $A_{13}$ and $A_{23}$.

Moreover, by increasing the values of quadratic nonlinearity in combination with parametric and external excitation one can observe additional peak in the response amplitude that appears due to the super-harmonic resonance (SHR). It can be also noticed that an increase of the quadratic nonlinearity parameter leads to an increase of both response amplitudes $A_{11}$ and $A_{21}$. The most prominent influence of the quadratic nonlinearity parameter is on the softening spring nonlinearity that occurs on SHR and primary resonance amplitudes. 
Furthermore, the unstable branches can be detected on response amplitudes for SHR as well, where multiple jumps effect also appears implying coexisting periodic orbits. On the other hand, the upper stable branch of the response amplitude becomes larger and wider, which is important feature for application in broadband EH systems.

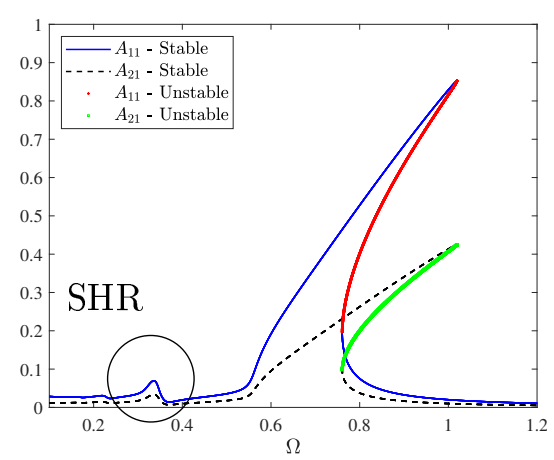

(a)

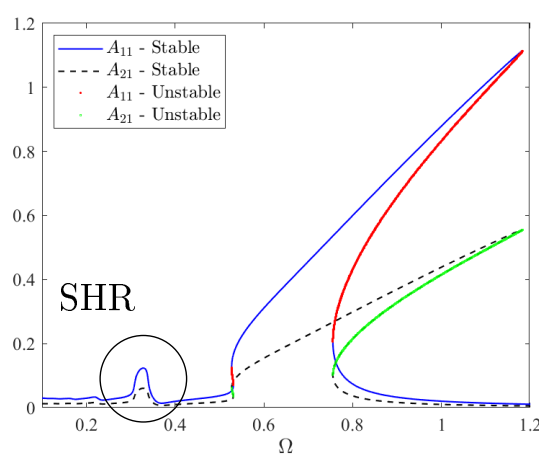

(b)

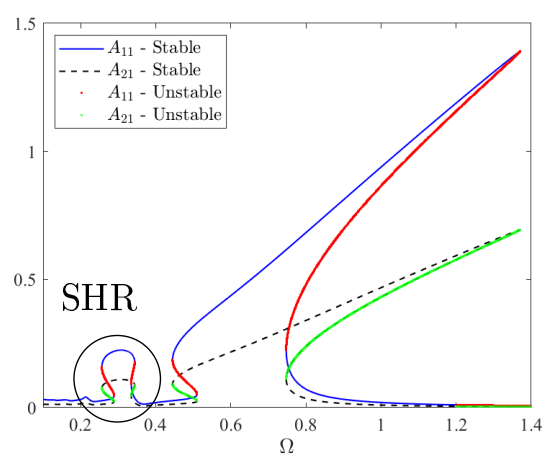

(c)

Figure 12: The amplitude-frequency response curves $A_{11}$ - blue solid line and $A_{21}$ - black dashed line for the nonlinear energy harvester with parametric excitation $p(\tau)=h \sin (\tau)$ and $h=0.3$. The stable periodic solutions are represented by the blue solid line for $A_{11}$ and dashed back line for $A_{21}$ while unstable periodic solutions are represented by the red line for $A_{11}$ and green solid line for $A_{21}$ and three values of quadratic nonlinearity: (a) $\xi=0.2$, (b) $\xi=0.4$, (c) $\xi=0.6$, (super-harmonic resonance SHR).

\section{Basins of attraction}

From above results it is obvious that the system is displaying the coexisting periodic solutions within certain frequency ranges due to mixed nonlinearities. Generally speaking, the amplitudes of coexisting periodic solutions depends on initial conditions as well as on starting positions of excitation frequency sweeping. This means that there are two sets of initial conditions for which the response amplitude after long time integration will converge i.e. towards the upper or lower branch of the periodic solution. This means that the system posses two attractors for the given set of material parameters and excitation frequencies. Marked in different colours for two sets of initial conditions, the response amplitude will converge to upper and lower branches forming the basin of attraction. For the presented nonlinear EH system, the basins of attraction are obtained for different values of the excitation frequency $\Omega$ as shown in Fig.13. Moreover, the coexisting periodic solutions from the amplitude-frequency responses given in Fig.10 are investigated based on varying initial conditions and three different values of excitation frequencies. To form the basin of attraction, the set of initial conditions $(x(0), \dot{x}(0))$ is given as a set of points in the phase space, where $400 \times 400$ is the number of adopted points. Setting the excitation frequency $\Omega=0.3$, the basin of attraction is given in Fig.13 (a). It can be noticed that the basin of attraction is displaying only one colour and therefore, there is only one stable periodic solution. Moreover, when increasing the excitation frequency $\Omega=0.8$ two coexisting periodic solutions appears as shown in Fig.13 (b). In this case, for the initial condition marked in red colour the solution converges to the upper stable branch while for the initial conditions marked in blue the solution converges to the lower stable branch. In Fig.10 one can observe that the excitation frequency $\Omega=0.8$ is close to the bifurcation point and therefore, for almost equal portions of initial conditions solution converges to the upper and lower stable branches of periodic solutions. However, further increase of the excitation frequency $\Omega=1$ leads to an increase of the set of initial conditions that converges to the lower stable branch of periodic solutions as shown in Fig.13 (c).

\section{Time response analysis}

To theoretically investigate efficiency of the proposed design og parametrically amplified EH system, the time responses of the displacement $x(\tau)$ and power $P(\tau)$ are plotted in Fig.14 and Fig.15. According to [34], the formulation of the dimensionless instantaneous power is given as $P(\tau)=\lambda v(\tau)^{2}$ and presented in the form of time response diagrams. By using the direct numerical integration (ode45 in Matlab), Eq.(3) and Eq.(4) are solved for different values of the magnitude of parametric excitation $h$ to show its effect on parametric amplification. To understand the influence of the parametric amplification on the initial harmonic excitation and performance of the $\mathrm{EH}$, the following initial conditions are adopted $x(0)=0.001, \dot{x}(0)=0.001, v(0)=0$ 


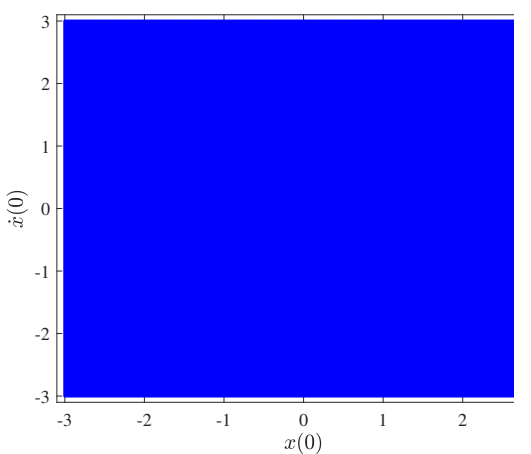

(a)

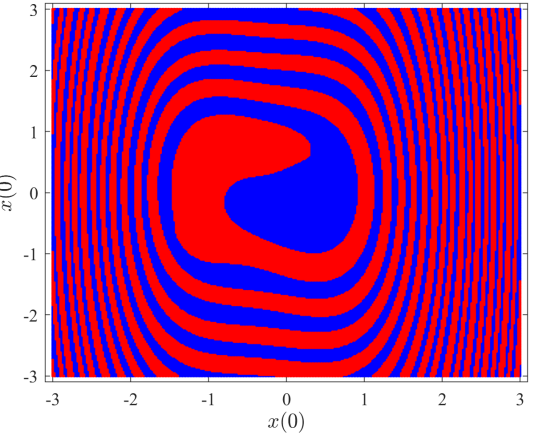

(b)

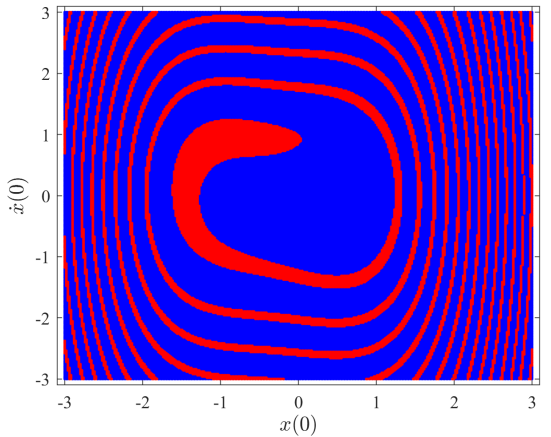

(c)

Figure 13: The basins of attraction for multiple coexisting periodic solutions given for three excitation frequencies and related to the amplitude-frequency response diagram given in Fig.10; (a) the excitation frequency $\Omega=0.3$, (b) the excitation frequency $\Omega=0.8$, and (c) the excitation frequency $\Omega=1$. The blue colour represents the set of initial conditions for which solution converges to the lower stable branch while the red colour represents the set of initial conditions for which the solution converges to the upper stable branch of periodic solutions.

in each of the presented numerical examples. Here, two cases are investigated with parametric excitations $p(\tau)=\sin (\tau)$ and $p(\tau)=\cos (2 \tau)$, where the following values of amplitudes $h=0,0.25,0.5$ are adopted. The dimensionless time period $T=200$ is used in all simulations.

The effects of varying parametric amplification $h$ on time responses of the displacement $x(\tau)$ and power $P(\tau)$ are presented in Fig.14 and Fig.15. One can observe that introduced parametric amplification increases the response amplitudes and power with respect to the harmonically excited case and thus having better performance in the energy harvesting application. It can be seen that small variations in the amplitude of parametric amplification $h$ results in higher response amplitudes of power $P(\tau)$. Also, after the first period of 50 time units, the influence of initial conditions becomes more prominent. After that period, the rest of the response is almost periodic for both types of parametric excitation, Fig.14. Moreover, Fig.15 shows changes in the instantaneous power response for two types of parametric excitation. One can observe that the effect of parametric amplification is the most pronounced for the value of parametric excitation amplitude $h=0.5$.

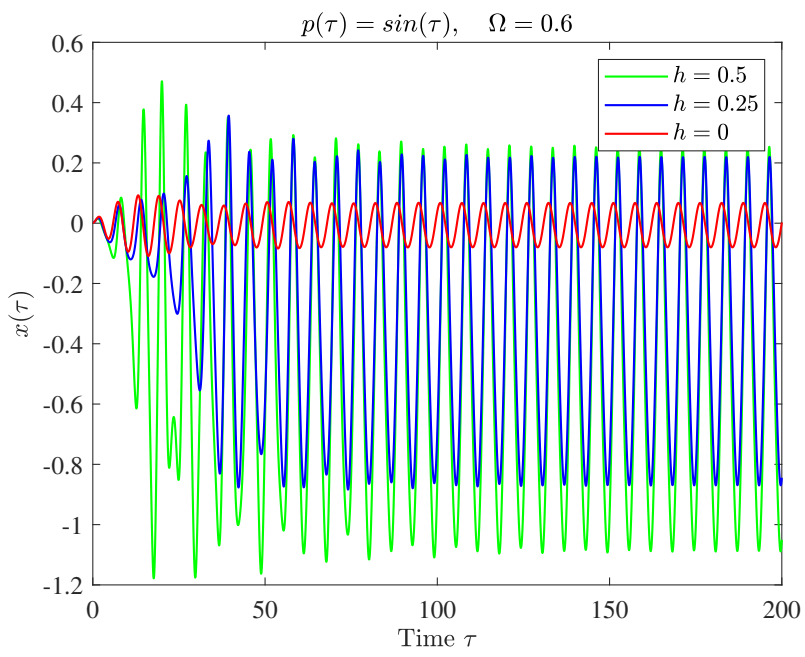

(a)

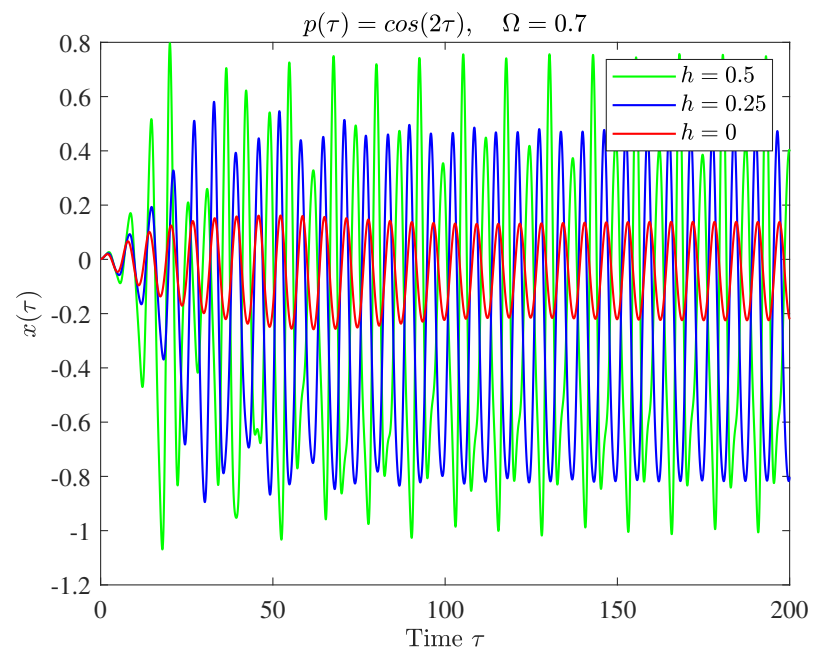

(b)

Figure 14: The time responses of the displacement $x(\tau)$ for time period $T=200$ and different values of the amplitude of parametric amplification $h=0,0.25,0.5$. The following sub-figures shows time response diagrams for $(\mathrm{a}) \sin (\tau)$ and $\Omega=0.6$ and (b) $\cos (2 \tau)$ and $\Omega=0.7$.

\subsection{Summary of the numerical results}

The following conclusions can be drawn about the results presented in this section. First of all, it is demonstrated that the introduction of parametric excitation into the nonlinear EH model based on Duffing oscillator significantly amplifies the harmonic response, which then leads to higher voltage responses and harvested power. Second, it is reviled that variation of the cubic nonlinearity term has a significant effect on the reduction of the softening property and unstable branches of periodic responses. Furthermore, an 


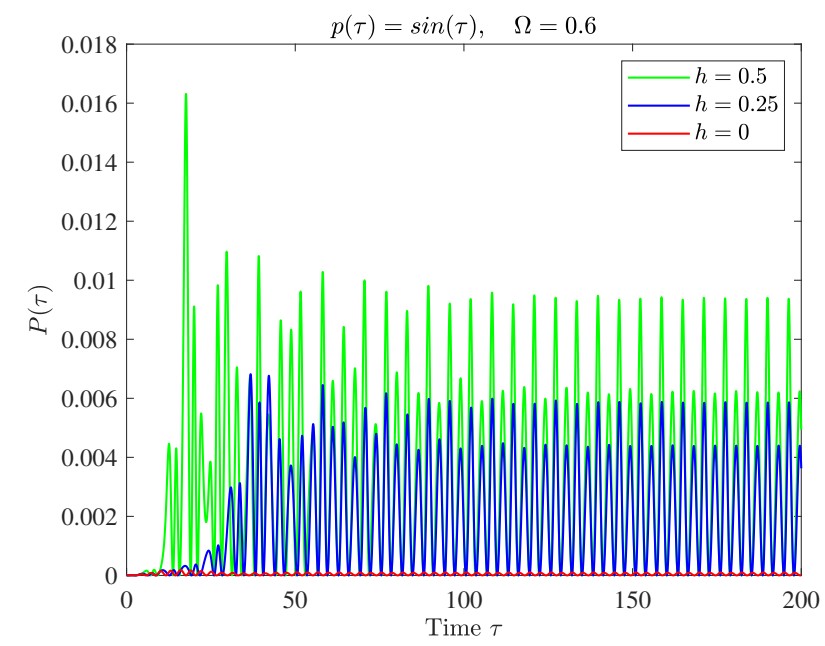

(a)

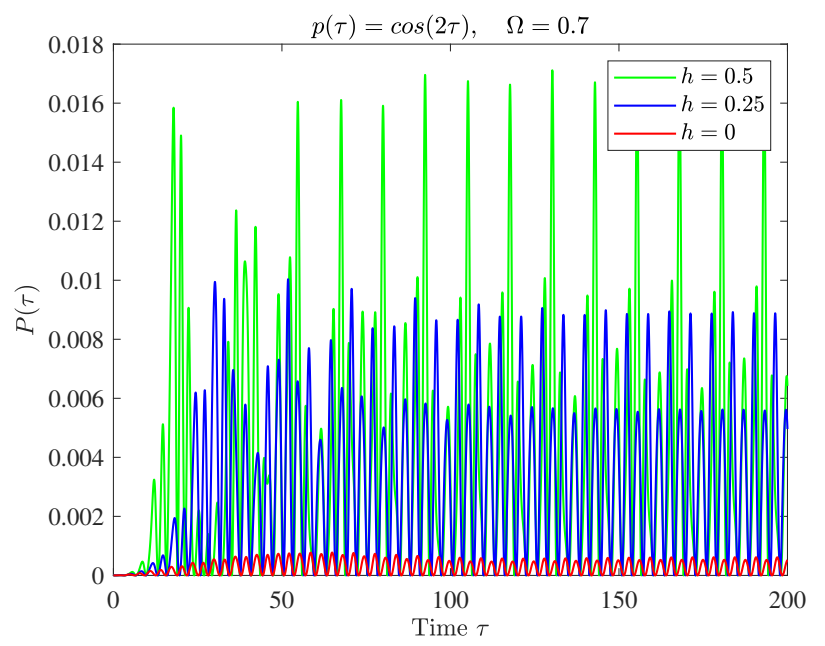

(b)

Figure 15: The instantaneous power $P=\lambda v^{2}(\tau)$ in time response diagrams determined for two types parametric amplification (a) $\sin (\tau)$ and $\Omega=0.6$, and (b) $\cos (2 \tau)$ and $\Omega=0.7$.

incremental change in the amplitude of parametric excitation leads to significant amplification of the response. The effect of this parameter is much more pronounced than change in any other parameter. Moreover, it is found that both softening and hardening spring effects become more prominent, and therefore the regions with coexisting periodic solutions expand. As expected, a change in quadratic nonlinearity leads to amplification of softening nonlinear property and appearance of additional unstable branches of periodic responses. Furthermore, it is observed that by considering the sine type of parametric excitation with one-to-one frequency tuning results in a significant amplification of the response amplitude. This kind of amplification appears in systems with combined nonlinearity, where influence of the quadratic nonlinearity term is significant for the existence of such effect. It is observed that one resonant super-harmonic peak appears before the primary resonant peak. This effect can be in the focus of some future studies of the broadband EH model. Due to the interesting results obtained from our theoretical investigation a future research would require an experimental analysis in order to realize a physical counterpart of the proposed EH system and further explore its dynamic behavior [28, 52].

\section{Conclusions}

The parametric amplification phenomenon is used to amplify the signal by direct harmonic excitation and enhance the performance of the broadband piezoelectric energy harvester. The forced Mathieu-Duffing oscillator model with quadratic nonlinearity and electric circuit equation is adopted to represent the observed energy harvester system. An approximation of the periodic response is obtained by using the incremental harmonic balance method in combination with the path-following to find the solution of the strongly nonlinear system. To obtain a complete periodic response, with upper and lower stable and unstable branches of periodic solutions, a Floquet stability theory and Hsu procedure are implemented. The results obtained by the proposed approach are verified with the direct numerical integration technique, where fine agreement is achieved.

In the numerical results section it is demonstrated that for a simultaneous action of the external harmonic excitation and parametric excitation, the system exhibits a combined resonant state i.e. the fundamental and parametric resonance state. For this case, it is shown that the response amplitude grows until it achieves its maximum value, which is governed by the introduced cubic nonlinearity. On the other hand, it is found that response amplitudes become larger for an increase of the value of quadratic nonlinearity term, especially for the system in combined resonant state. Furthermore, it was shown that the cosine form of parametric excitation term results in much higher response amplitudes compared to the case with the sinusoidal form. Moreover, an incremental change of parametric excitation magnitude has significant effect on softening and hardening spring nonlinearity.

Analysis of the amplitude-frequency responses have shown that the first three amplitudes are having the most of the system's energy, which is related to the order of nonlinearity that appears in the system. A wide spectrum of nonlinear phenomena is detected such as softening and hardening spring nonlinearity, multiple jump-up and jump-down phenomena, which indicates the existence of coexisting periodic solutions. 
It can be outlined that the parametric excitation in conjunction with the direct harmonic excitation magnify the response amplitude several times with respect to the the case with only external harmonic excitation. Time response diagrams of the instantaneous power additionally revealed the advantages of parametric amplification in improving the energy harvesting capabilities of the proposed EH system. These findings can be important in future applications and potential development of more efficient broadband piezoelectric energy harvesters. The presented methodology can be implemented in the analyses of more complex EH models, especially for systems with a higher number of degrees of freedom and combined nonlinearities. Moreover, the parametric amplification phenomena can be used to amplify a signal detected in the directly excited system and therefore increase the effective Q-factor for sensing applications in MEMS and NEMS devices.

\section{Acknowledgements}

D. Karličić and S. Adhikari were supported by the Marie Skłodowska-Curie Actions - European Commission: 799201-METACTIVE. M. Cajić was sponsored by the Serbian Ministry of Education, Science and Technological Development and Mathematical institute of the Serbian Academy of Sciences and Art.

\section{References}

[1] Alper Erturk and Daniel J Inman. Piezoelectric energy harvesting. John Wiley \& Sons, 2011.

[2] Shashank Priya, Hyun-Cheol Song, Yuan Zhou, Ronnie Varghese, Anuj Chopra, Sang-Gook Kim, Isaku Kanno, Liao Wu, Dong Sam Ha, Jungho Ryu, et al. A review on piezoelectric energy harvesting: materials, methods, and circuits. Energy Harvesting and Systems, 4(1):3-39, 2019.

[3] G Gatti, MJ Brennan, MG Tehrani, and DJ Thompson. Harvesting energy from the vibration of a passing train using a single-degree-of-freedom oscillator. Mechanical Systems and Signal Processing, 66:785-792, 2016.

[4] Xiangyang Li, Deepesh Upadrashta, Kaiping Yu, and Yaowen Yang. Analytical modeling and validation of multi-mode piezoelectric energy harvester. Mechanical Systems and Signal Processing, 124:613-631, 2019.

[5] Samuel C Stanton, Clark C McGehee, and Brian P Mann. Nonlinear dynamics for broadband energy harvesting: Investigation of a bistable piezoelectric inertial generator. Physica D: Nonlinear Phenomena, 239(10):640-653, 2010.

[6] Roszaidi Ramlan, MJ Brennan, BR Mace, and I Kovacic. Potential benefits of a non-linear stiffness in an energy harvesting device. Nonlinear dynamics, 59(4):545-558, 2010.

[7] Mohammed F Daqaq, Ravindra Masana, Alper Erturk, and D Dane Quinn. On the role of nonlinearities in vibratory energy harvesting: a critical review and discussion. Applied Mechanics Reviews, 66(4), 2014.

[8] Sergio P Pellegrini, Nima Tolou, Mark Schenk, and Just L Herder. Bistable vibration energy harvesters: a review. Journal of Intelligent Material Systems and Structures, 24(11):1303-1312, 2013.

[9] Thomas Huguet, Mickaël Lallart, and Adrien Badel. Orbit jump in bistable energy harvesters through buckling level modification. Mechanical Systems and Signal Processing, 128:202-215, 2019.

[10] Ivana Kovacic and Michael J Brennan. The Duffing equation: nonlinear oscillators and their behaviour. John Wiley \& Sons, 2011.

[11] Junyi Cao, Arkadiusz Syta, Grzegorz Litak, Shengxi Zhou, Daniel J Inman, and Yangquan Chen. Regular and chaotic vibration in a piezoelectric energy harvester with fractional damping. The European Physical Journal Plus, 130(6):103, 2015.

[12] Michael I Friswell, Onur Bilgen, S Faruque Ali, Grzegorz Litak, and Sondipon Adhikari. The effect of noise on the response of a vertical cantilever beam energy harvester. ZAMM-Journal of Applied Mathematics and Mechanics/Zeitschrift für Angewandte Mathematik und Mechanik, 95(5):433-443, 2015.

[13] DX Cao, S Leadenham, and A Erturk. Internal resonance for nonlinear vibration energy harvesting. The European Physical Journal Special Topics, 224(14-15):2867-2880, 2015.

[14] Wei Yang and Shahrzad Towfighian. Internal resonance and low frequency vibration energy harvesting. Smart Materials and Structures, 26(9):095008, 2017.

[15] Yu Jia and Ashwin A Seshia. An auto-parametrically excited vibration energy harvester. Sensors and Actuators A: Physical, 220:69-75, 2014.

[16] P Brzeski, E Pavlovskaia, T Kapitaniak, and P Perlikowski. The application of inerter in tuned mass absorber. International Journal of Non-Linear Mechanics, 70:20-29, 2015.

[17] Antonio SE Chong, Yuan Yue, Ekaterina Pavlovskaia, and Marian Wiercigroch. Global dynamics of a harmonically excited oscillator with a play: Numerical studies. International Journal of Non-Linear Mechanics, 94:98-108, 2017.

[18] Shengxi Zhou, Junyi Cao, Daniel J Inman, Jing Lin, Shengsheng Liu, and Zezhou Wang. Broadband tristable energy harvester: modeling and experiment verification. Applied Energy, 133:33-39, 2014.

[19] Meghashyam Panyam and Mohammed F Daqaq. Characterizing the effective bandwidth of tri-stable energy harvesters. Journal of Sound and Vibration, 386:336-358, 2017.

[20] Shengxi Zhou and Lei Zuo. Nonlinear dynamic analysis of asymmetric tristable energy harvesters for enhanced energy harvesting. Communications in Nonlinear Science and Numerical Simulation, 61:271-284, 2018.

[21] Pilkee Kim and Jongwon Seok. A multi-stable energy harvester: Dynamic modeling and bifurcation analysis. Journal of Sound and Vibration, 333(21):5525-5547, 2014. 
[22] Khondokar Billah. On the definition of parametric excitation for vibration problems. Journal of sound and vibration, 270(1-2):450-454, 2004.

[23] Ivana Kovacic, Richard Rand, and Si Mohamed Sah. Mathieu's equation and its generalizations: Overview of stability charts and their features. Applied Mechanics Reviews, 70(2):020802, 2018.

[24] Andrew N Cleland. Thermomechanical noise limits on parametric sensing with nanomechanical resonators. New Journal of Physics, 7(1):235, 2005.

[25] Jeffrey F Rhoads and Steven W Shaw. The impact of nonlinearity on degenerate parametric amplifiers. Applied Physics Letters, 96(23):234101, 2010.

[26] Jeffrey F Rhoads, Nicholas J Miller, Steven W Shaw, and Brian F Feeny. Mechanical domain parametric amplification. Journal of Vibration and Acoustics, 130(6):061006, 2008.

[27] Stefan Neumeyer, VS Sorokin, and Jon Juel Thomsen. Effects of quadratic and cubic nonlinearities on a perfectly tuned parametric amplifier. Journal of Sound and Vibration, 386:327-335, 2017.

[28] Stefan Neumeyer, VS Sorokin, MHM van Gastel, and Jon Juel Thomsen. Frequency detuning effects for a parametric amplifier. Journal of Sound and Vibration, 445:77-87, 2019.

[29] Vladislav S Sorokin. On the unlimited gain of a nonlinear parametric amplifier. Mechanics Research Communications, 62:111-116, 2014.

[30] Amit Dolev and Izhak Bucher. Tuneable, non-degenerated, nonlinear, parametrically-excited amplifier. Journal of Sound and Vibration, 361:176-189, 2016.

[31] A Dolev and I Bucher. Dual frequency parametric excitation of a nonlinear, multi degree of freedom mechanical amplifier with electronically modified topology. Journal of Sound and Vibration, 419:420-435, 2018.

[32] Amit Dolev and Izhak Bucher. Optimizing the dynamical behavior of a dual-frequency parametric amplifier with quadratic and cubic nonlinearities. Nonlinear Dynamics, 92(4):1955-1974, 2018.

[33] Wei Yang and Shahrzad Towfighian. A parametric resonator with low threshold excitation for vibration energy harvesting. Journal of Sound and Vibration, 446:129-143, 2019.

[34] Mohamed Belhaq, Zakaria Ghouli, and Mustapha Hamdi. Energy harvesting in a mathieu-van der pol-duffing mems device using time delay. Nonlinear Dynamics, 94(4):2537-2546, 2018.

[35] Yu Jia. Review of nonlinear vibration energy harvesting: Duffing, bistability, parametric, stochastic and others. Journal of Intelligent Material Systems and Structures, 23(21):1-24, 2020.

[36] Yu Jia, Jize Yan, Kenichi Soga, and Ashwin A Seshia. A parametrically excited vibration energy harvester. Journal of intelligent material systems and structures, 25(3):278-289, 2014.

[37] MF Daqaq and D Bode. Exploring the parametric amplification phenomenon for energy harvesting. Proceedings of the Institution of Mechanical Engineers, Part I: Journal of Systems and Control Engineering, 225(4):456-466, 2011.

[38] Panagiotis Alevras, Stephanos Theodossiades, and Homer Rahnejat. Broadband energy harvesting from parametric vibrations of a class of nonlinear mathieu systems. Applied Physics Letters, 110(23):233901, 2017.

[39] Miguel Gonzalez and Yoonseok Lee. A study on parametric amplification in a piezoelectric mems device. Micromachines, 10(1):19, 2019.

[40] Daniel Platz and Ulrich Schmid. Vibrational modes in mems resonators. Journal of Micromechanics and Microengineering, 29(12):123001, 2019.

[41] Hanna Cho, Min-Feng Yu, Alexander F Vakakis, Lawrence A Bergman, and D Michael McFarland. Tunable, broadband nonlinear nanomechanical resonator. Nano letters, 10(5):1793-1798, 2010.

[42] James M Lehto Miller, Azadeh Ansari, David B Heinz, Yunhan Chen, Ian B Flader, Dongsuk D Shin, L Guillermo Villanueva, and Thomas W Kenny. Effective quality factor tuning mechanisms in micromechanical resonators. Applied Physics Reviews, 5(4):041307, 2018.

[43] Hanna Cho, Bongwon Jeong, Min-Feng Yu, Alexander F Vakakis, D Michael McFarland, and Lawrence A Bergman. Nonlinear hardening and softening resonances in micromechanical cantilever-nanotube systems originated from nanoscale geometric nonlinearities. International Journal of Solids and Structures, 49(15-16):2059-2065, 2012.

[44] Gyan Prakash, Arvind Raman, Jeffrey Rhoads, and Ronald G Reifenberger. Parametric noise squeezing and parametric resonance of microcantilevers in air and liquid environments. Review of Scientific Instruments, 83(6):065109, 2012.

[45] Jon Juel Thomsen. Vibrations and stability: advanced theory, analysis, and tools. Springer Science \& Business Media, 2003.

[46] YK Cheung, SH Chen, and S_L Lau. Application of the incremental harmonic balance method to cubic non-linearity systems. Journal of Sound and Vibration, 140(2):273-286, 1990.

[47] JH Shen, KC Lin, SH Chen, and KY Sze. Bifurcation and route-to-chaos analyses for mathieu-duffing oscillator by the incremental harmonic balance method. Nonlinear Dynamics, 52(4):403-414, 2008.

[48] JL Huang, RKL Su, YY Lee, and SH Chen. Nonlinear vibration of a curved beam under uniform base harmonic excitation with quadratic and cubic nonlinearities. Journal of Sound and Vibration, 330(21):5151-5164, 2011.

[49] Udbhau Bhattiprolu, Anil K Bajaj, and Patricia Davies. Periodic response predictions of beams on nonlinear and viscoelastic unilateral foundations using incremental harmonic balance method. International Journal of Solids and Structures, 99:28-39, 2016.

[50] Sheng Wang, Lin Hua, Can Yang, Xinghui Han, and Zhuoyu Su. Applications of incremental harmonic balance method combined with equivalent piecewise linearization on vibrations of nonlinear stiffness systems. Journal of Sound and Vibration, 441:111-125, 2019.

[51] CS Hsu. On approximating a general linear periodic system. Journal of Mathematical Analysis and Applications, 45(1):234251, 1974.

[52] Vijay Kumar, Jacob K Miller, and Jeffrey F Rhoads. Nonlinear parametric amplification and attenuation in a base-excited cantilever beam. Journal of sound and vibration, 330(22):5401-5409, 2011. 